\title{
Self-Organization Properties of CSMA/CA Systems and Their Consequences on Fairness
}

\author{
Mathilde Durvy, Student Member, IEEE, Olivier Dousse, Member, IEEE, and Patrick Thiran, Member, IEEE
}

\begin{abstract}
Decentralized medium access control schemes for wireless networks based on CSMA/CA, such as the IEEE 802.11 protocol, are known to be unfair. In multihop networks, they can even favor some links to such an extent that the others suffer from virtually complete starvation. This observation has been reported in quite a few works, but the factors causing it are still not well understood. We find that the capture effect and the relative values of the receive and carrier sensing ranges play a crucial role in the performance of these protocols. Using a simple Markovian model, we show that an idealized CSMA/CA protocol suffers from starvation when the receiving and sensing ranges are equal, but quite surprisingly that this unfairness is reduced or even disappears when these two ranges are sufficiently different. We also show that starvation has a positive counterpart, namely organization. When its access intensity is large the protocol organizes the transmissions in space in such a way that it maximizes the number of concurrent successful transmissions. We obtain exact formulæ for the so-called spatial reuse of the protocol on large line networks.
\end{abstract}

Index Terms-Ad hoc networks, CSMA/CA, medium-access control (MAC), modeling, multihop, performance analysis.

\section{INTRODUCTION}

$\mathbf{T}$ HE IEEE 802.11 [1] medium-access control (MAC) protocol is unfair. This statement has been so often repeated that it is now widely accepted. Indeed, in single-hop networks where all nodes can communicate directly, it has been shown that the Binary Exponential Backoff (BEB) of the 802.11 protocol creates situations where a single node has an almost exclusive access to the communication channel. In multihop networks, similar starvation phenomena have been reported ([2]-[6]). However, contrary to the single-hop case, very few models are able to explain the starvation problems encountered in multihop topologies. Existing models often concentrate on very small network topologies or describe how a specific feature of the 802.11 protocol affects its fairness. In addition, it is common to assume that the receiving and carrier sensing ranges are equal, or to neglect the the so-called "capture effect" and to assume instead that two packets received at the same

Manuscript received December 05, 2007; revised November 19, 2008. Current version published February 13, 2009. The work was supported in part by the National Competence Center in Research on Mobile Information and Communication Systems (NCCR-MICS), a center supported by the Swiss National Science Foundation under Grant 5005-67322.

M. Durvy and P. Thiran are with EPFL, CH-1015 Lausanne, Switzerland (e-mail: mathilde.durvy@epfl.ch; patrick.thiran@epfl.ch).

O. Dousse is with Nokia Research Center, CH-1015 Lausanne, Switzerland (e-mail: olivier.dousse@nokia.com).

Communicated by E. Modiano, Associate Editor for Communication Networks.

Digital Object Identifier 10.1109/TIT.2008.2011427 time always collide. We elaborate on existing models and their assumptions in Section II.

The goal of this work is to shed some light on how such assumptions affect the performance of general CSMA/CA protocols. Our approach is the following. Instead of concentrating on specific implementation aspects, we consider an idealized protocol that retains the key features of CSMA/CA protocols such as their carrier sensing, collision avoidance, and backoff mechanisms. Section III describes this protocol and the metrics used to evaluate its performance. We use continuous time Markov chains to describe the dynamics of the protocol. We find that even though this idealized protocol does not suffer from the well-known problems that have already been identified in single-hop and multihop networks, it is still subject to the starvation phenomenon described in the literature. However, we observe that the starvation phenomenon results from an optimal organization of the transmissions in space. Indeed, at high access intensities the idealized protocol maximizes, in a completely decentralized way, the number of simultaneous successful transmissions in the network. This is quite surprising as the problem of finding the transmission patterns that maximize spatial reuse in a general network is NP-Complete (by equivalence with the maximal independent set problem [7]). Following the work of [8], [9] and [10] have recently proposed several distributed algorithms to approach this goal ${ }^{1}$. Yet, we also show that the performance of the protocol are very sensitive to the assumptions made in terms of carrier sensing range and capture effect.

The main contribution of this paper is the explanation of the starvation and the organization phenomenons. In particular, we show under which condition these phenomenons occur and why. We separate our analysis into three classes of assumptions, each leading to a different Markov chain structure. Section IV covers the simplest case, that has previously been studied in the literature. It includes known results together with our own results. The purpose of this section is to keep the paper self-contained and to serve as a reference case for the study in the two next sections.

In Sections V and VI, we develop new Markov models to address the cases where the nodes' receiving range and carrier sensing range are significantly different. These two sections differ in the underlying assumptions on the nodes' capture capability, and consequently in the structure of the Markov models involved. In particular, in terms of their reversibility.

Finally, the Appendix contains a lemma used to derive exact formulæ for the performance of the idealized protocol under a simple line network topology.

\footnotetext{
${ }^{1}$ In fact, they consider a slightly more general problem, the maximal weight independent set problem.
} 


\section{RELATED WORK}

For a long time, models for the 802.11 protocol have been limited to the single-hop setting and numerous works have extended the original papers of Bianchi [11] and Cali, Conti, and Gregori [12], [13]. Similarly, the capture effect has first been studied for slotted Aloha schemes [14]-[16], and more recently for single-hop 802.11 networks [17], [18]. Unfortunately, models for the 802.11 protocol in the single-hop setting are not easy to extend to the multihop setting, because they rely explicitly on the central assumption that all nodes can hear each other and that there are no hidden terminals. Lately however, several papers have attempted to model the behavior of CSMA/CA protocols in the multihop setting. This section is dedicated to such papers, as our work is devoted to CSMA/CA multihop networks. In particular, we concentrate on their ability to characterize the spatial reuse/fairness performance of these protocols. We also try to highlight the assumptions made in terms of carrier sensing and capture models.

[19] makes a first step towards a multihop analysis of the 802.11 protocol. Their model accounts for the capture effect but is valid only for a limited number of hidden terminals. [20] and [21] are really the first to consider the operation of the 802.11 protocol on a real multihop network. Their models either perform a fixed point iteration or use numerical methods to obtain the throughput performance of the 802.11 protocol on specific network topologies (a ring for [20] and a Poisson topology for [21]). The work of [2] forms the basis our paper. It extends the Markov chain formalism used by [22], [23] to model the CSMA protocol to an idealized CSMA/CA protocol. The strength of this model is to preserve the dependence between nodes, which is typical of CSMA and CSMA/CA protocols. Using this model, [2] is probably the first to predict and explain the starvation phenomenon on general multihop topologies. The ability of CSMA/CA protocols to reach a high spatial reuse or throughput when all nodes of a multihop networks are saturated has been highlighted in [3], [24], and [25]. [26] studies the throughput region using mean-field techniques, which are valid in the limit when the number of nodes within the same interference range is arbitrary large but allow to include adaptive back-offs. However, all these works ([20], [21], [3], [2], [24]-[26]) consider only the case where the receiving and carrier sensing ranges are equal, and do not take the capture effect into account.

[4]-[6], [27], [28], and [29] relax the assumption of equal receiving and carrier sensing ranges but still neglect the capture effect. These papers present results in terms of throughput and fairness, but only the models of [5] and [6] are able to predict the starvation phenomenon. The work of [6] does not investigate the effect of the carrier sensing range on the performance of the 802.11 protocol, it rather concentrates on the role played by the minimum contention window in the starvation phenomenon. [27], [28], and [29] concentrate on the end-to-end throughput of a single flow or path. [5] uses a 50 node 2-D topology on a $1000 \times 1000 \mathrm{~m}$ square area to validate its model. The receiving range is set to $200 \mathrm{~m}$ and the carrier sensing range to either 200 or $400 \mathrm{~m}$. [5] observes that the unfairness of the 802.11 protocol

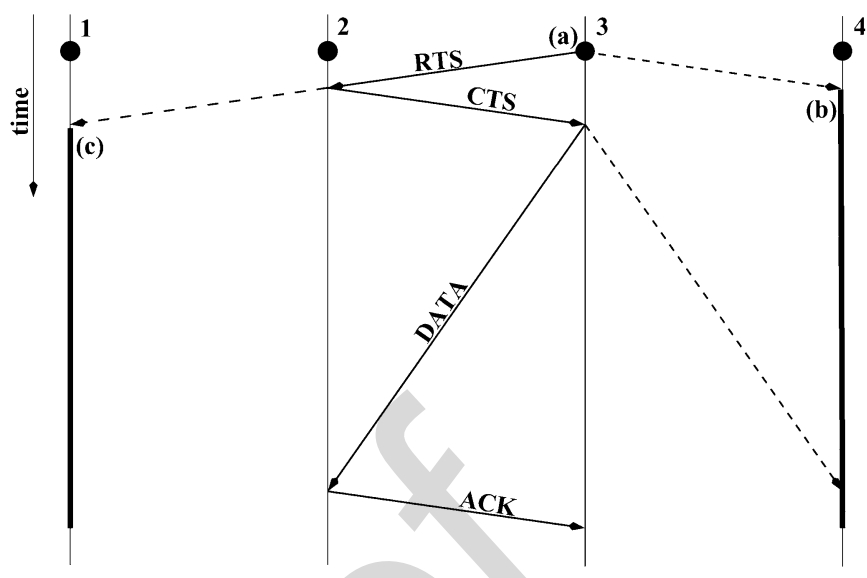

Fig. 1. A typical exchange between Node 3 and Node 2. At point (a) the backoff timer of Node 3 reaches zero, Node 3 sends a RTS packet to Node 2. Upon receiving the RTS (b) (respectively CTS (c)) Node 4 (respectively Node 1) sets its NAV (bold line) to cover the duration of the exchange between Node 3 and Node 2.

is higher at a large carrier sensing range. Although the model of [4] does not fully predict the starvation phenomenon, the authors also observe it by simulation. Interestingly, they use the same experimental setting as [5] (with different node positions), but their simulations show that the unfairness of the 802.11 protocol is now higher at a small carrier sensing range. These apparently contradicting results are probably a consequence of the limited amount of multihopping possible in the topology used in [5] and [4] (indeed, the receiving and carrier sensing ranges are such that they cover a large part of the network).

In this paper, we recognize the need to consider larger network topologies with reduced border effects to obtain more predictable results. We extend the work of [2] to 1) allow for different receive and carrier sensing ranges, 2) account for the capture effect, and 3) provide closed-form expressions for the spatial reuse of CSMA/CA protocols on large line networks. We favor the model of [2] over the other models as it makes the starvation phenomenon directly apparent in its equations.

Part of this work has been presented in the conference papers [3] and [30].

\section{FRAMEWORK}

\section{A. Medium-Access Control (MAC) Layer}

1) Protocol Overview: We first provide a high-level overview of the CSMA/CA protocol that we analyze.

In this protocol, a node intending to transmit senses first the medium. Physical and virtual carrier sensing mechanisms are used to determine the state of the medium. The physical carrier sensing is provided by the physical layer. The virtual carrier sensing is done through an RTS/CTS handshake, as illustrated in Fig. 1: The sender first sends a "request to send" packet (RTS), to which the receiver answers with a "clear to send" packet (CTS). Both packets contain information about the time at which the exchange will be completed, so that overhearing nodes can update their network allocation vector (NAV) and refrain from emitting during the exchange. In this way, interferences from other transmissions are avoided. 


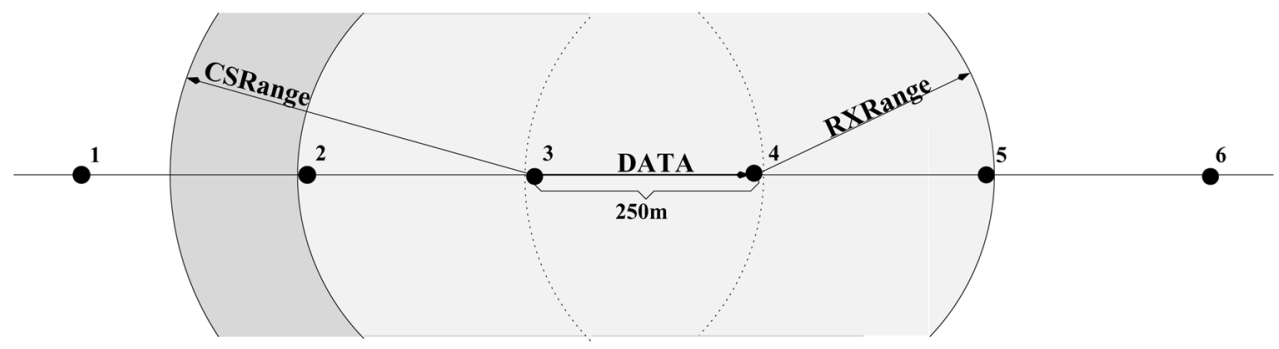

Fig. 2. All links with an end-node in the gray domain around the active link are silenced (nine directed links are thus silenced). As the nodes in the transmitter's CSRange are in its RXRange, the exclusion domain is symmetric.

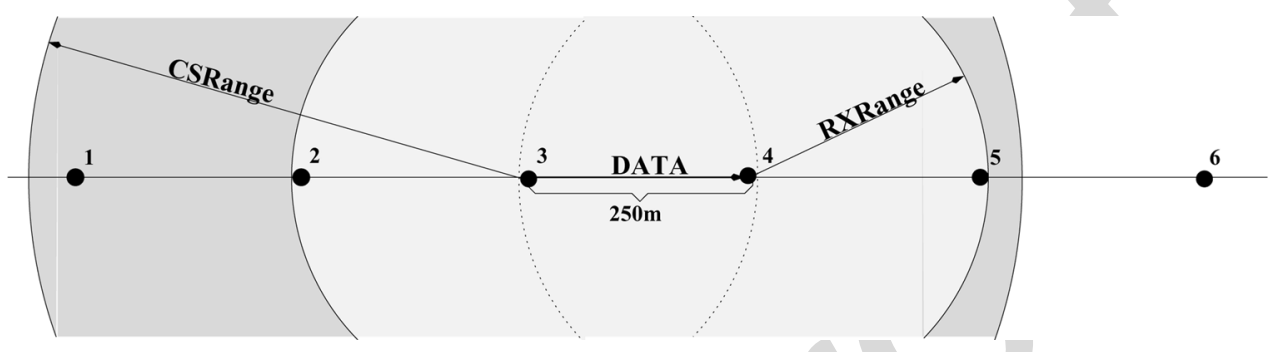

Fig. 3. As the CSRange is much larger than the RXRange, more nodes are silenced around the transmitter (Node 3). The exclusion domain is asymmetric.

However, this mechanism has the drawback of synchronizing transmission attempts: If several nodes have been waiting to transmit due to an ongoing transmission, they will attempt to access the channel as soon as the transmission completes (i.e., as soon as they sense no activity on the channel). This creates predictable collisions. To avoid this problem, each node maintains a backoff timer, which is initialized to a random value chosen according to some backoff distribution. Timers run when the channel is idle; when a node senses the channel busy, it temporarily freezes its timer. Nodes may start emitting only when their timer reaches zero. After each transmission, the emitter resets its timer to a new random value. This mechanism causes random delays between the end of a transmission and the start of the next one, and spreads transmission attempts in time.

2) Assumptions: In this paper, we consider an idealized CSMA/CA protocol in order to capture the essential features of CSMA/CA systems, and leave aside the effects due to the imperfection of real protocols (we refer the reader to [31] for an overview of those effects in IEEE 802.11).

First, we assume that the RTS/CTS handshake is so short that its duration can be ignored in the model. In addition, we consider a continuous backoff distribution, instead of the discrete distribution implemented in actual protocols, so that two timers have a zero probability to expire at the same time. These two assumptions imply that no collision occurs due to simultaneous transmission attempts.

We also assume that nodes maintain a separate backoff timer for each of their outgoing links, as recommended by [32] in an attempt to guarantee per link fairness at least in the context of single-hop networks.

3) Interaction With the Physical Layer: We model the physical layer using three parameters: the receiving range, the carrier sensing range and the interference range.

- The receiving range (RXRange) is the maximum distance from the source at which a packet can be successfully re- ceived in the absence of interfering nodes. In practice, the RXRange depends on the rate at which a packet is sent. In our model, we assume that all packets (including RTS and CTS packets) are sent with the same rate, so that the same ranges apply to any transmission. We say that there is a link from the source to each of the nodes in its RXRange.

- The carrier sensing range (CSRange) is the maximum distance from the emitter at which its transmission can still be detected, but not necessarily decoded. In this way, all nodes within the CSRange of an emitter sense the channel busy during its transmission. The CSRange is always larger than or equal to the RXRange.

After the initial RTS-CTS handshake, nodes in the RXRanges of the sender and the receiver are silenced by their virtual carrier sensing mechanism. In addition, nodes within CSRange of the sender are kept from sending by their physical carrier sensing mechanism. We refer to the domain silenced around an active link by its virtual and physical carrier sensing as its exclusion domain. In this work we distinguish between symmetric exclusion domains (Fig. 2, Section IV) and asymmetric exclusion domains (Fig. 3, Sections V and VI).

- The interference range (IRange) is the maximum distance from the receiver at which an interferer can prevent the successful decoding of a transmission. Unlike the other two ranges, the interference range follows from a SINR constraint at the receiver and depends on the actual distance between the emitter and the receiver. The IRange is the largest when this distance is equal to the RXRange, and we denote this maximal interference range by IRange*.

4) Collisions: A collision is the failure of a packet transmission due to the interference from another concurrent transmission. In our framework, collisions are avoided if the protocol successfully prevents all nodes in the interference range of the 
receiver to start a transmission. In the sequel, we will assume that it is the case. In other words, we assume that any node in the interference range of a receiver is also in the exclusion domain of the receiver's link. This happens if

$$
\text { RXRange } \geq \text { IRange* }
$$

or if

$$
\text { CSRange } \geq \text { IRange }^{*}+\text { RXRange }
$$

or of course if no node happens to lie in the interference range of the receiver (for example because of a regular placement of the nodes).

5) Capture Effect: However, the above assumption does not completely prevent overlapping transmissions: Assume that a node in the receiver's CSRange but outside of the link's exclusion domain (for example Node 6 in Fig. 3) starts to transmit. As the two nodes (Node 4 and Node 6 in the example) are within CSRange of each other, the new transmission (from Node 6) can be sensed at the receiver (Node 4) and thus interferes with the ongoing transmission (from Node 3 to 4 ). However, because of the assumption (1), the new transmitter (Node 6) is necessarily outside the receiver's IRange, so that the interference is not strong enough to create a collision. The signal-to-interference ratio at the receiver (Node 4) is still high enough for successful decoding the ongoing transmission and the new interfering signal is simply ignored: this is called the capture effect. In this work, we will consider two different models for capture effects, described, respectively, in Sections V-A and VI-A.

\section{B. Metrics}

Denote by $L$ the number of node pairs that can communicate (i.e., are in receiving range of each other). There are thus $2 L$ directed links in the network. We use two metrics to characterize the performance of our protocol.

1) Average Spatial Reuse $(\sigma)$ : We define the average spatial reuse as the number of active links normalized by $L$ (and averaged over time) in the network.

2) Fairness Index (FI): To assess the MAC layer fairness of the protocol we use Jain's Fairness Index [33]. Denote by $p(j)$ the probability that link $j$ be active under a given medium access control protocol. The link fairness index of the protocol is

$$
\mathrm{FI}=\frac{\left(\sum_{j} p(j)\right)^{2}}{2 L \sum_{j} p(j)^{2}} .
$$

The maximum fairness index is 1 . It corresponds to a network where all links access the channel equally. Yet, if only $k$ of the directed links have an equal access to the channel and the remaining links have no access to the channel, the fairness index is $k /(2 L)$.

\section{Problem Setting}

Our analysis assumes saturated traffic conditions (i.e., nodes always have a packet to send to each of their one-hop neighbors) and, unless otherwise specified, we consider exponentially distributed backoff and exchange times.

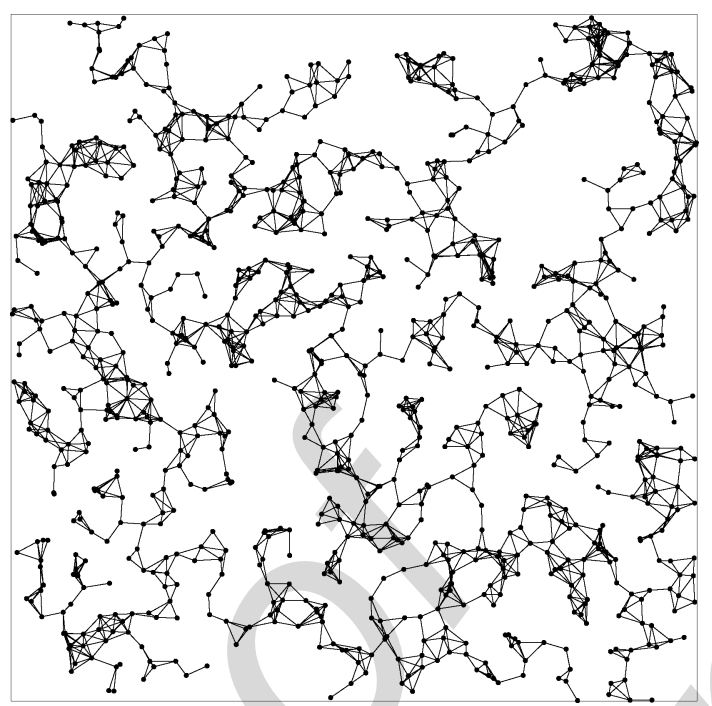

Fig. 4. Random 2-D topology.

All simulations in this paper were performed using a dedicated event driven simulator for our idealized protocol (code available at [34]). We simulated two different network topologies: a line network topology (50 equally spaced nodes at a distance of $250 \mathrm{~m}$ ) and a random 2-D topology (1065 nodes are randomly deployed on a $6500 \times 6500$ area, the isolated nodes are then removed to keep a connected component of 970 nodes, the average node degree is approximately 5 (Fig. 4)). In both topologies, the RXRange is $250 \mathrm{~m}$. For symmetric exclusion domains, the IRange* and the CSRange are equal to $250 \mathrm{~m}$ as well. For asymmetric exclusion domains, the IRange* is $445 \mathrm{~m}$ and the CSRange is equal to $550 \mathrm{~m}$ for the line and to $695 \mathrm{~m}$ for the random 2-D topology (this is necessary to make the protocol collision free). The simulations correspond to approximately 15 $\mathrm{min}$ of traffic. To provide accurate results, each simulation is repeated 20 times (using different random seeds). The presented results consist of the values averaged over the 20 experiments and, of the $95 \%$ confidence intervals.

\section{THe SyMmetric CASE}

\section{A. Specific Assumptions}

The exclusion domain around a link is symmetric if the set of nodes silenced by it is the same as the set silenced by the reverse link (the link with permuted emitter and receiver). In our physical layer model, this happens if no node is located outside the RXRange of the transmitter while being located inside its CSRange.

Exclusion domains are always symmetric if the CSRange and the RXRange are equal. However, symmetric exclusion domains often arise when the network topology is regular (see example in Fig. 2). For example, indoor environments with their carefully designed network topologies and strong signal attenuation may lead to such a situation.

The case with symmetric exclusion domain has already been studied in [2] using a Markov model very similar to the one presented below, which is a class of loss networks [35]. This section 


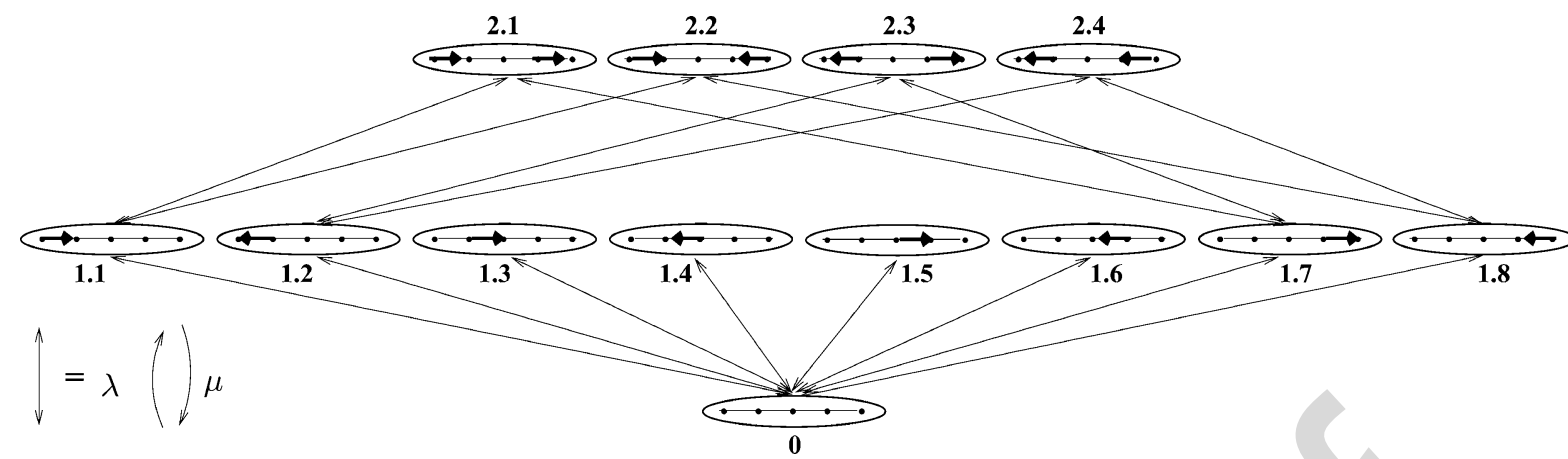

level 2

$N(2)=4$ no active link, $N(1)=8$ states with 1 active link, and $N(2)=4$ states with 2 active links.

extends the results of [2] by looking at a specific topology: the line topology. In particular, we obtain a closed-form formula for the spatial reuse of the protocol on large line networks (Theorem $1)$.

\section{B. Markov Modeling}

The dynamics of our idealized protocol can be described by a continuous Markov chain. At any given time, a set of directional links is active in the network. Such a set is called a transmission pattern and defines a state of the Markov chain. Clearly, only patterns that respect the constraints set by the exclusion domains of active links are possible. Hence the set of all valid patterns forms the state space of the Markov chain. We say that a state is at level $i$ if the corresponding transmission pattern has exactly $i$ active links, and we denote by $N(i)$ the number of such states. The states at the highest level are called patterns of maximal spatial reuse: they will be of particular interest for the analysis of the Markov chain.

Transitions in the Markov chain can only occur between states that are separated by one level: A transition between a state at level $i$ and a state at level $i-1$ corresponds to the completion of a transmission, and a transition between a state at level $i$ and a state at level $i+1$ corresponds to the beginning of a new transmission (which is of course possible only if the new link does not lie in the exclusion domain of already active links).

Denote by $\mu^{-1}$ the average exchange time and by $\lambda^{-1}$ the average backoff time. The transition rate between a state at level $i$ and a state at level $i-1$ (respectively, at level $i+1$ ) is $\mu$ (resp. $\lambda$ ). Fig. 5 gives an example of such a Markov chain. Finally, we define the access intensity as $\rho:=\lambda / \mu$.

The stationary distribution of this reversible Markov chain can be computed using the global balance equations and has a product form. Indeed, the stationary probability of any state at level $i$ is equal to

$$
\pi(i)=\rho^{i} \pi(0)
$$

where $\pi(0)$ is the stationary probability of the empty transmission pattern which is equal to

$$
\pi(0)=\left(\sum_{i} N(i) \rho^{i}\right)^{-1} .
$$

This form of stationary distributions first appeared in the context of circuit-switched loss networks [35] and it is known [36] that (2) and (3) are insensitive to the exchange time distribution. The average spatial reuse is then

$$
\sigma=\frac{1}{L} \sum_{i} i N(i) \pi(i)
$$

where $L$ is the number of undirected links in the network. To derive the long-term fairness of the protocol, we also need to compute the probability that a specific link is active. A link $j$ is active if the chain is in a state whose transmission pattern contains link $j$. Denote by $N(i, j)$ the number of such patterns with a total number of active links equal to $i$. The probability $p(j)$ that link $j$ is active is

$$
p(j)=\sum_{i} N(i, j) \pi(i)
$$

and the fairness index can be computed using the definition of Section III-B2.

For $\rho>1, \pi(i)$ increases with the value of $i$, and the transmission patterns with a high number of active links have an increased probability of appearing, compared to those with only a few active links. In the limit $\rho \rightarrow \infty$, only the transmission patterns that maximize the spatial reuse have a nontrivial stationary probability. Consequently, when the average backoff time is much lower than the average exchange time, the idealized protocol achieves the maximal spatial reuse. However, all links that do not belong to the transmission patterns of maximal spatial reuse are starved.

The Line Topology: Equations (2) through (5) are valid for any network topology. Unfortunately, it is in general not possible to obtain a closed form expression for $N(i)$ nor $N(i, j)$. However, we can do so for a line topology, where $L+1$ nodes (numbered from 0 to $L$ ) are equally spaced (by 1 space unit) along a straight line (Fig. 6).

We define the parameter $l$ as 1 plus the minimal distance separating two noncolliding transmissions. This means that there can be an active link every $l$ space units. For example, if the RXRange and the CSRange cover exactly one neighbor, transmissions can take place simultaneously every 3 space units (see Fig. 6), so that $l=3$ in this case. Therefore, finding possible transmission patterns boils down to packing segments of length $l$ in a line interval of length $L+(l-1)$ (the additional term 


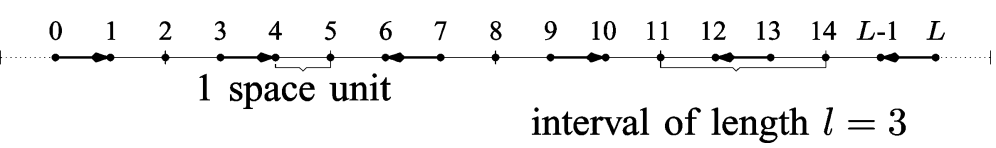

Fig. 6. A transmission pattern of maximal spatial reuse with symmetric exclusion domains (both RXRange and CSRange covering one neighbor).

$(l-1)$ compensates for the border effects, and is illustrated by the dashed segments in Fig. 6). If we place $i$ such segments, there remains $v=L-(l-1)-i l$ vacant units in the interval. The number of ways one can dispose these $i$ segments is thus equal to $i+v$ choose $i$. Finally, as each segment of length $l$ correspond to two possible link activations in the network (due to the symmetry assumption, each link and its reverse link occupy the same space), each possible arrangement of $i$ segments corresponds to $2^{i}$ possible link activation patterns, and we get

$$
N(i)=2^{i}\left(\begin{array}{c}
i+v \\
i
\end{array}\right)
$$

Next, we count the number of valid transmission patterns with $i$ active links that include a given link. We denote by $j_{\text {right }}$ the link between node $j-1$ and node $j$, and by $j_{\text {left }}$ the link between node $j$ and node $j-1$. To compute $N\left(i, j_{\text {right }}\right)$, we have to count the number of ways to place $i-1$ active links around the already active link $j_{\text {right }}$. Assume that we place $k$ of these active links on the left of link $j_{\text {right }}$, and $(i-1)-k$ on its right. We obtain

$$
\begin{aligned}
N\left(i, j_{\text {right }}\right)=2^{i-1} \sum_{k=0}^{i-1}\left(\begin{array}{c}
k+v_{\text {left }} \\
k
\end{array}\right) & \\
& \times\left(\begin{array}{c}
(i-1)-k+v_{\text {right }} \\
(i-1)-k
\end{array}\right)
\end{aligned}
$$

where $v_{\text {left }}=j-1-l k$ and $v_{\text {right }}=L-j-l((i-1)-k)=$ $L+l-j-l(i-k)$. Clearly, as the exclusion domain of link $j_{\text {right }}$ and link $j_{\text {left }}$ are identical by assumption, the computation of $N\left(i, j_{\text {left }}\right)$ yields the same result. By (5), this implies that, in the symmetric setting, two links in opposite directions have the same probability to be active.

Plugging the expressions for $N(i)$ and $N(i, j)=$ $N\left(i, j_{\text {right }}\right)=N\left(i, j_{\text {left }}\right)$ in (2) through (5) we can compute analytically the spatial reuse and the fairness index of the protocol on a finite line topology. Note that these values depend on the total length of the network $L$.

Theorem 1: For large line networks, i.e., when $L \rightarrow \infty$, the average spatial reuse $\sigma$ of the protocol under symmetric exclusion domains converges to

$$
\lim _{L \rightarrow \infty} \sigma=\frac{2 \rho y_{1}^{l-1}}{1+2 l \rho y_{1}^{l-1}}
$$

where $y_{1}$ is the positive real root of $1-y-2 \rho y^{l}$.

Proof: The result is obtained by applying Lemma 1 (in the Appendix) with $k=\left\lfloor\frac{L+(l-1)}{l}\right\rfloor, m=l, r=2 \rho$ and $n=$ $(L+(l-1)) \bmod l$. We then divide by $l$ to obtain the average spatial reuse $\sigma$.

Alternatively, this theorem can also be shown by adapting the approach of [37] which uses multivariate generating functions combined with the residue method. Although this approach yields the same result, we believe our approach is simpler.
Moreover, Lemma 1 can also be used to obtain the average spatial reuse under asymmetric exclusion domains, as we will see in the next section. As expected, when $\rho$ increases, $\sigma$ tends to $1 / l$, which is the maximal spatial reuse.

\section{Simulation Results}

The plain curves of Figs. 7 and 8 show the performance of the idealized protocol on the 50 node line network topology described in Section III-C ${ }^{2}$ with symmetric exclusion domains. Our analytical predictions and the values obtained by simulation differ by at most $0.2 \%$. Figs. 7 and 8 clearly illustrate the trade-off between spatial reuse and fairness. As $\rho$ increases (i.e., as the average backoff time becomes small compared to the average exchange time), the spatial reuse of the protocol reaches the maximal spatial reuse of 0.3469 (the value is slightly above $1 / 3$ because of the border effects) but the fairness decreases. Fig. 9(a) gives the probability that a link is active during the simulation. We see that only the links that belong to the pattern of maximal spatial reuse have a good access to the channel. In fact, our analysis tells us that in the limit, when $\rho \rightarrow \infty$, only these links have a nontrivial probability of accessing the channel: This means that, like the spatial reuse, the fairness index converges to 0.3469 . To summarize, under symmetric exclusion domains, a large fraction of the links ( $\simeq 2 / 3$ in our case) can be completely starved ${ }^{3}$.

\section{The Asymmetric Case With Full Capture EfFect}

\section{A. Specific Assumptions}

The exclusion domain around a link is asymmetric if there are nodes inside the transmitter's CSRange that are outside its RXRange (as Node 1 in Fig. 3). In our model, these nodes are unable to decode the RTS packets from the transmitter and are silenced only through the physical carrier sensing. Therefore, more nodes are silenced on the transmitter's side, and this is why we call the exclusion domain asymmetric.

As described in Section III-A4, if the set of nodes covered by the emitter's RXRange and CSRange differs, transmissions may overlap and create a capture effect. In this section, we assume that capture occurs whenever a node receives concurrently a strong signal from an emitter in its RXRange and a weaker signal from another emitter in its CSRange (but not in its RXRange). In particular, we do not take the order of arrival of the signals into account: even if the strongest signal begins after the weakest one, the receiver is able to synchronize on the strongest signal (see Fig. 10 for an example). This is a rather optimistic scenario, although it is supported to some extent by

\footnotetext{
${ }^{2}$ The impatient reader will have to wait until Section VI to discover the results on the 2-D topology as the line topology makes it easier to visualize and explain the behavior of the protocol.

${ }^{3}$ More detailed simulation studies under this setting have been performed in [3]. These studies show in particular that the above observations remain valid for other backoff and exchange time distributions.
} 


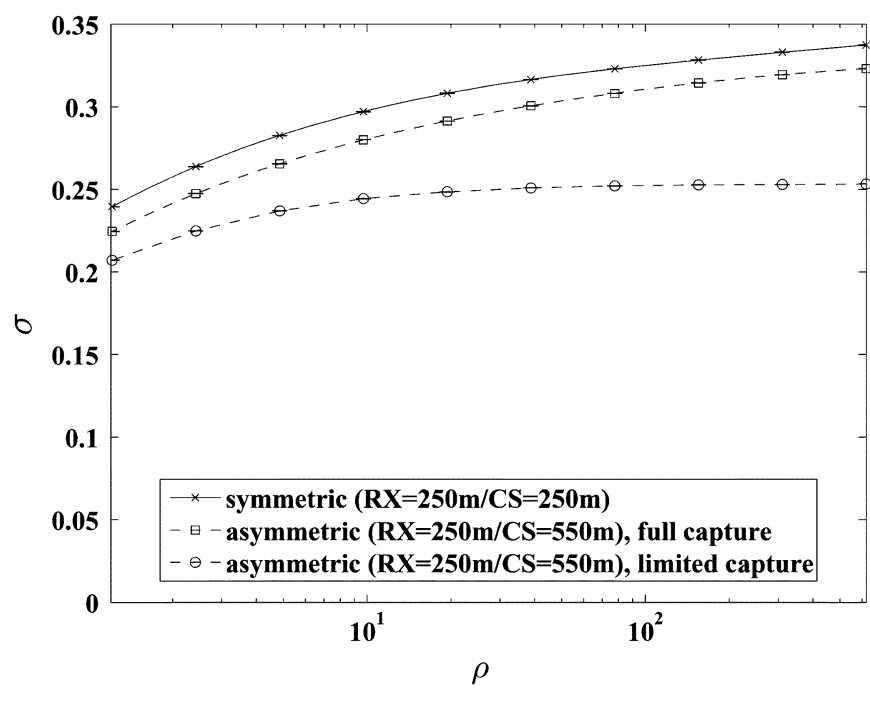

Fig. 7. Spatial reuse achieved by the idealized protocol on a line topology of 50 nodes, as a function of the access intensity $\rho$. The markers correspond to the results obtained by simulations and the curves to the results obtained analytically (except for the 'asymmetric $(\mathrm{RX}=250 \mathrm{~m} / \mathrm{CS}=550 \mathrm{~m})$, limited capture' case where we do not have the exact analytical curve).

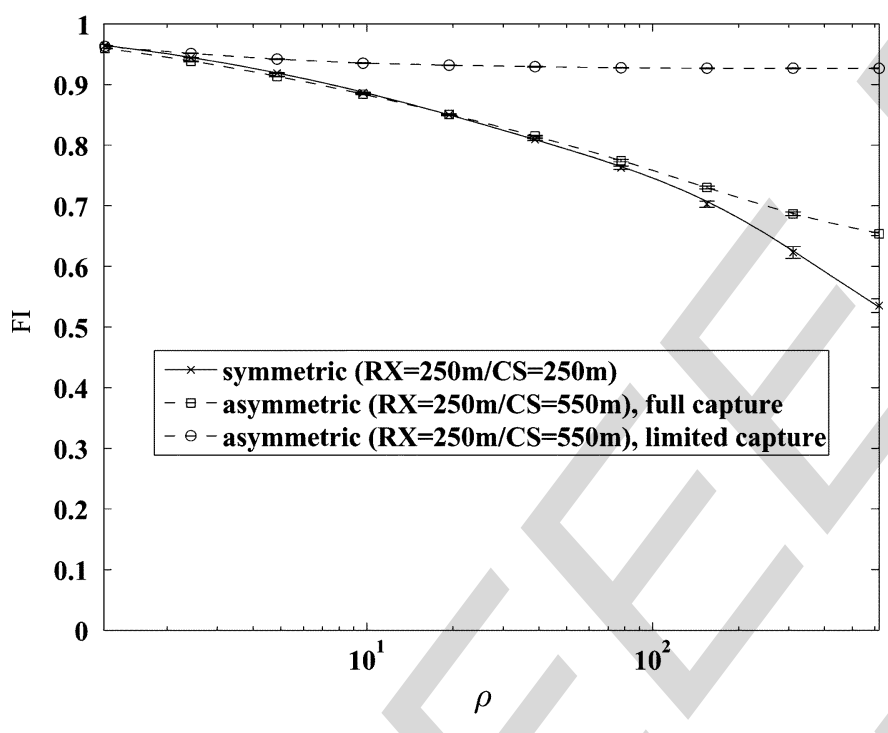

Fig. 8. Fairness index of the idealized protocol on a line topology of 50 nodes. The format is the same as in Fig. 7.

experimental evidences. [38] reports that capture occurs even if the strongest packet arrives later, but within the physical preamble of the first packet. In essence, full capture means that the order of arrival of the transmissions does not matter and it implies that patterns are valid independently of the order of arrival of the transmissions. Note that this last assumption will be removed in Section VI.

As an example, consider again a line network topology where nodes are equally spaced and the RXRange covers one neighbor, but where the CSRange covers two neighbors. A transmission pattern is valid if two consecutive receivers are separated by at least 2 space units, and if two consecutive transmitters are separated by at least 3 space units (see Fig. 11). Under this setting, the maximal spatial reuse is still $1 / 3$, but it is not possible to

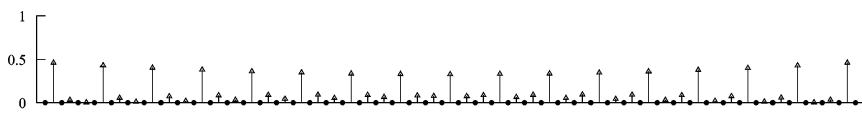

(a)

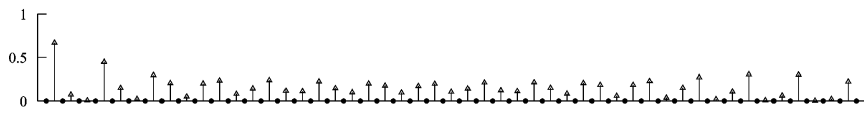

(b)

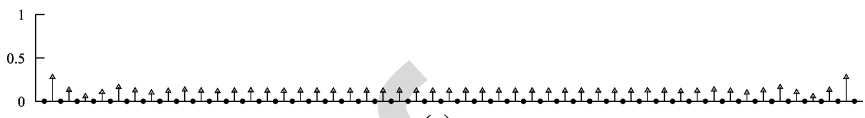

(c)

Fig. 9. On the $x$ axis, a line topology of 50 nodes. To each link $j_{\text {right }}$ (going from left to right) we associate a vertical bar that corresponds to the probability $p\left(j_{\text {right }}\right)$ that the link be active in the simulation for $\rho=620$ (the $p\left(j_{\text {left }}\right)$ can be obtained flipping the graphs). (a) Symmetric exclusion domain (RXRange $=$ CSRange $=250 \mathrm{~m}$ ). $\sigma=0.34, \mathrm{FI}=0.53$. (b) Asymmetric exclusion domains (RXRange $=250 \mathrm{~m}$, CSRange $=550 \mathrm{~m})$ with full capture. $\sigma=0.32, \mathrm{FI}=0.65$. (c) Asymmetric exclusion domains (RXRange $=$ $250 \mathrm{~m}$, CSRange $=550 \mathrm{~m}$ ) with limited capture. $\sigma=0.25, \mathrm{FI}=0.93$.

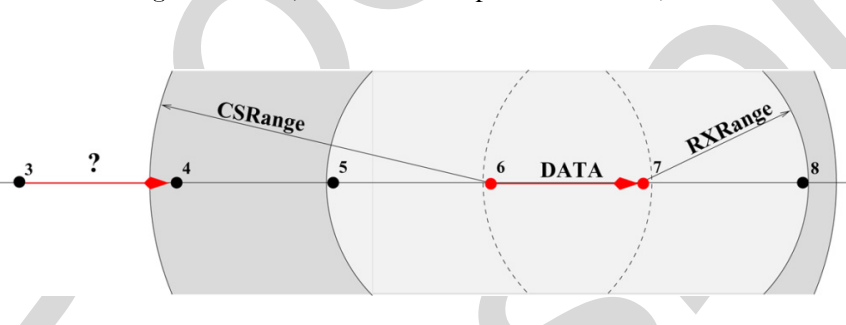

Fig. 10. Assume there is an ongoing DATA transmission between Node 6 and Node 7. As Node 4 is in the CSRange of Node 6, it can detect this transmission. If Node 3 sends a RTS to Node 4 we have two possibilities. Either Node 4 can resynchronize on this stronger signal, in which case the RTS will be followed by a complete exchange between Node 3 and Node 4; or it cannot resynchronize on the strongest signal and the RTS will be lost. In the full capture model, we always assume the first possibility whereas in the the limited capture model, the second possibility is assumed. Note that in the full capture model, a node that is silenced only through its physical carrier sensing (such as Node 4) can play the role of a receiver but it cannot initiate an exchange.

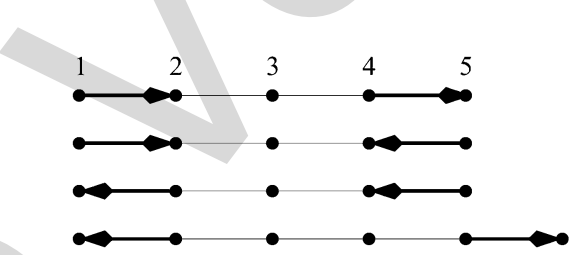

Fig. 11. Among the four possible configurations of two active links placed as closely to each other as possible (with $l=3$ ), only the three first ones maximize spatial reuse.

have two transmitters back to back in a pattern of maximal spatial reuse.

\section{B. Markov Modeling}

Asymmetric exclusion domains and capture effects make the analytical study of the CSMA/CA protocols more challenging and no model has been proposed so far. We now show that the Markovian framework of the previous section can be extended to include this case. The structure of the Markov chain remains essentially unchanged but some states (and the corresponding transitions) disappear. Take for example the Markov chain of Fig. 5 and assume that the CSRange now covers two neighbors instead of one. All transmission patterns remain valid except the 


\section{Direction change}

Fig. 12. A transmission pattern of maximal spatial reuse (with $l=3$ ). Active links are sorted in two groups, inside which all links point in the same direction.

\section{Vacant space}

Fig. 13. A transmission pattern with one vacant space (with $l=3$ ). There are three points where links change direction.

pattern 2.3 at the top level of the Markov chain, where two transmitters are back to back. This change in the set of valid transmission patterns does not affect the reversibility of the Markov chain. Equations (2) through (5) still hold, and the asymptotic properties of the protocol for $\rho \rightarrow \infty$ are thus unchanged. However, the number $N(i)$ of valid transmission patterns with $i$ active links and the number $N(i, j)$ of such patterns including link $j$ have to be recomputed. Again, this is only tractable for the simple topologies. We compute these numbers explicitly for the line topology in the following paragraph.

The Line Topology: We begin with the observation that two consecutive active links along the line can be separated by the least number of space units if and only if their orientation is such that the two transmitters are not back to back (see Fig. 11). Again, to each active link we associate an interval of length $l$ on the line. Consider a transmission pattern of maximal spatial reuse and assume that, once we have placed the intervals corresponding to its active links, there is no vacant space ${ }^{4}$; because of the above observation, all active links must point in the same direction until a certain point, and then point to the other direction (see Fig. 12). If $i$ is the number of active links, there are clearly $i+1$ possible settings. Now, let us assume that there is one vacant space. This vacant space will allow for an additional change of direction, as depicted in Fig. 13. Therefore, in this case, there is a total of three points where links change direction and there are

$$
\left(\begin{array}{c}
i+3 \\
3
\end{array}\right)=\left(\begin{array}{c}
i+3 \\
i
\end{array}\right)
$$

ways to pick these three points 5 . This reasoning can be extended to an arbitrary number of vacant spaces $v$, and we find

$$
N(i)=\left(\begin{array}{c}
i+2 v+1 \\
i
\end{array}\right)
$$

where $v=L+(l-1)-l i$.

To obtain an expression for $N(i, j)$ we follow the same procedure as in Section IV-B. If link $j_{\text {right }}$ from node $j-1$ to node $j$ is active, we enumerate the possible ways to place $k$ active links on its left side and $(i-1)-k$ active links on its right side. On the right side, the problem consists simply in packing $(i-1)-k$ intervals of length $l$ in an interval of length $L-j$. On the left

\footnotetext{
${ }^{4}$ Note that this is only possible for certain values of line size $L$.

${ }^{5}$ Note that these points can be collocated. Two collocated points correspond to a vacant space without a change of direction. Three collocated points correspond to a vacant space and two receivers facing each other. The problem is equivalent to counting the number of 3 -combinations with repetition of an $(i+1)$-element
} set. side, the problem is slightly trickier, as node $j-l$ cannot emit, which means that in our previous reasoning we can do one less change of direction. We obtain

$$
\left(\begin{array}{c}
k+2 v_{\text {left }} \\
k
\end{array}\right)
$$

possible settings only, where $v_{\text {left }}=j-1-l k$. Combining the left and right parts, we get

$$
\begin{aligned}
& N\left(i, j_{\text {right }}\right)=\sum_{k=0}^{i-1}\left(\begin{array}{c}
k+2 v_{\text {left }} \\
k
\end{array}\right) \\
& \times\left(\begin{array}{c}
(i-1)-k+2 v_{\text {right }}+1 \\
(i-1)-k
\end{array}\right)
\end{aligned}
$$

where $v_{\text {right }}=L-j-l((i-1)-k)=L+l-j-l(i-k)$. The same reasoning allows to compute the value of $N\left(i, j_{\text {left }}\right)$ :

$$
\begin{aligned}
N\left(i, j_{\text {left }}\right)=\sum_{k=0}^{i-1}\left(\begin{array}{c}
k+2 v_{\text {left }}+1 \\
k
\end{array}\right) & \\
& \times\left(\begin{array}{c}
(i-1)-k+2 v_{\text {right }} \\
(i-1)-k
\end{array}\right) .
\end{aligned}
$$

Note that in the asymmetric case with full capture links $j_{\text {left }}$ and $j_{\text {right }}$ do not necessarily have the same probability to be active.

We can then plug the expressions for $N(i)$ and $N(i, j)$ in (2)(3)(4) through (5) to obtain the exact curve for the spatial reuse and the fairness index of the protocol.

The asymptotic spatial reuse for large networks is obtained in a similar way as in the case of symmetric exclusion domains.

Theorem 2: For line networks with $L \rightarrow \infty$, the average spatial reuse $\sigma$ of the protocol under asymmetric exclusion domains and full capture converges to

$$
\lim _{L \rightarrow \infty} \sigma=\frac{2 \rho y_{1}^{2 l-1}}{1+2 l \rho y_{1}^{2 l-1}}
$$

where $y_{1}$ is the positive real root of $1-y-\rho y^{2 l}$.

Proof: The result is obtained by applying Lemma 1 (in the Appendix) with $k=\left\lfloor\frac{L+(l-1)}{l}\right\rfloor, m=2 l, r=\rho$ and $n=$ $2((L+(l-1)) \bmod l)+1$. We then divide by $l$ to obtain the average spatial reuse $\sigma$.

Again, we verify that $\sigma$ tends indeed to $1 / l$ when $\rho$ tends to infinity.

\section{Simulation Results}

We illustrate by simulation the influence of asymmetric exclusion domains on the performance of the idealized protocol. Fig. 7 shows that its spatial reuse increases less rapidly than in the symmetric case. At a given $\rho$, the protocol with asymmetric exclusion domains has therefore on average fewer active links than in the symmetric case. This has a positive effect on the fairness of the protocol, especially at high values of $\rho$, where operating with a slightly lower number of active links greatly improves the fairness. Fig. 9(b) confirms that the starvation phenomenon is less pronounced than in the symmetric case and that the links in the middle of the line topology obtain a fairer access to the channel. Yet, we also see that the distribution of 
the link activity is not symmetric anymore. This is in agreement with our analysis that tells us that two links in opposite directions do not necessarily have the same probability of accessing the channel. This effect has a negative impact on the fairness index of the protocol. We have therefore two competing effects. Fig. 8 shows that, at low values of $\rho$, the two effects compensate each others; the fairness index of the protocol is very similar under symmetric and asymmetric exclusion domains. However, at high values of $\rho$, the positive effect dominates; the fairness index of the protocol in the asymmetric case with full capture is above its fairness index in the symmetric case. In the limit, when $\rho \rightarrow \infty$, the behavior of the protocol under asymmetric exclusion domains with full capture is essentially the same as under symmetric exclusion domains. The spatial reuse goes to 0.3469 and approximately $2 / 3$ of the links get starved. The only difference is that under asymmetric exclusion domains some links belong to more patterns of maximal spatial reuse as others, depending on their direction: this reduces the FI from 0.3469 to 0.2676 .

\section{The Asymmetric CASE With Limited CAPTURE EFFECT}

\section{A. Specific Assumptions}

In this section, we consider asymmetric exclusion domains with limited instead of full capture. Limited capture means that the capture effect occurs only if the strongest signal comes first. This assumption reflects the fact that in practice, many radio circuits cannot resynchronize to a stronger signal if they are already locked on another (weaker) carrier. Consequently, in this capture model, the order of arrival of the transmissions does matter.

Consider the example in Fig. 10. The transmission from Nodes 6 to 7 begins first. Node 4 senses this first transmission and locks its synchronization circuit on it. When Node 3 sends a RTS packet to initiate a transmission, Node 4 is unable to resynchronize and decode it. The RTS packet is lost and no transmission follows. As a result, in Fig. 11, the first (top) case is possible only if the left most link becomes active first (the opposite is true for the third case).

The limited capture effect is implemented in the most wellknown network simulators, including Ns-2 and Qualnet.

\section{B. Simulation Results}

These simulation results were at first quite surprising to us. Figs. 7 and 8 show the performance of the idealized protocol with asymmetric exclusion domains and limited capture. The spatial reuse of the protocol increases to a value much below $1 / 3$ but the fairness index of the protocol remains above 0.9, even for large values of $\rho$. The access probabilities of the different links presented in Fig. 9(c) illustrate even better the dramatic improvement of the protocol fairness at high access intensity. Except at the borders, the situation is now completely fair. These results are good news in practice: They show that the starvation phenomenon is fixed by receivers having a limited capture capability, without requiring any other modification to the protocol.

To consolidate our findings, we show by simulation that this observation also holds in a random two-dimensional topology.

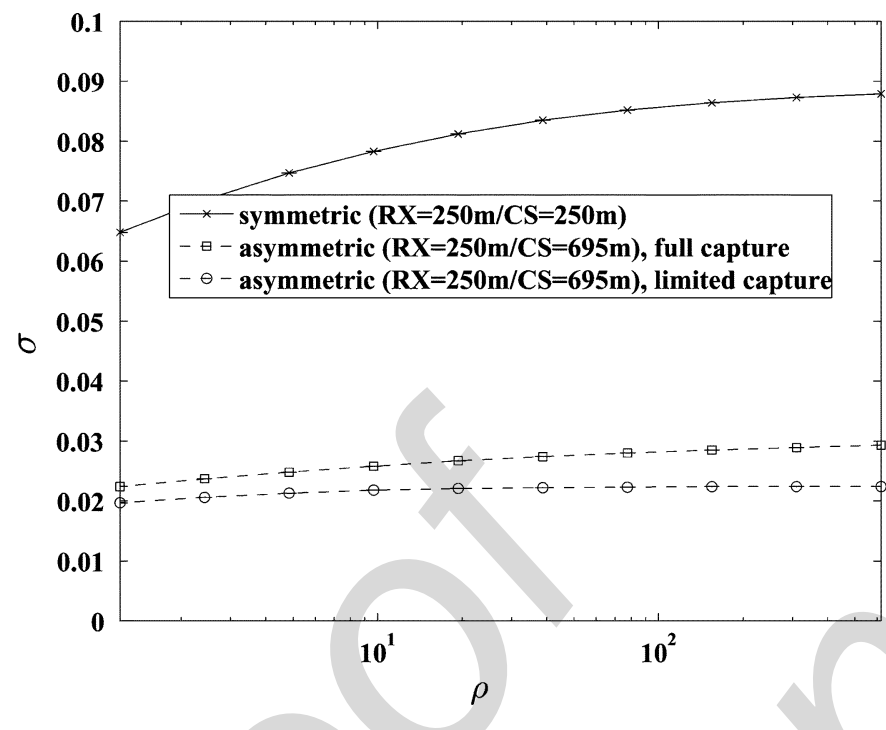

Fig. 14. Spatial reuse achieved by the idealized protocol on the 2-D topology (simulation results). The average spatial reuse is computed using the definition of Section III-B1 with $L=2435$. In this particular topology, at most 217 links can be activated simultaneously, yielding a maximal spacial reuse of 0.089 .

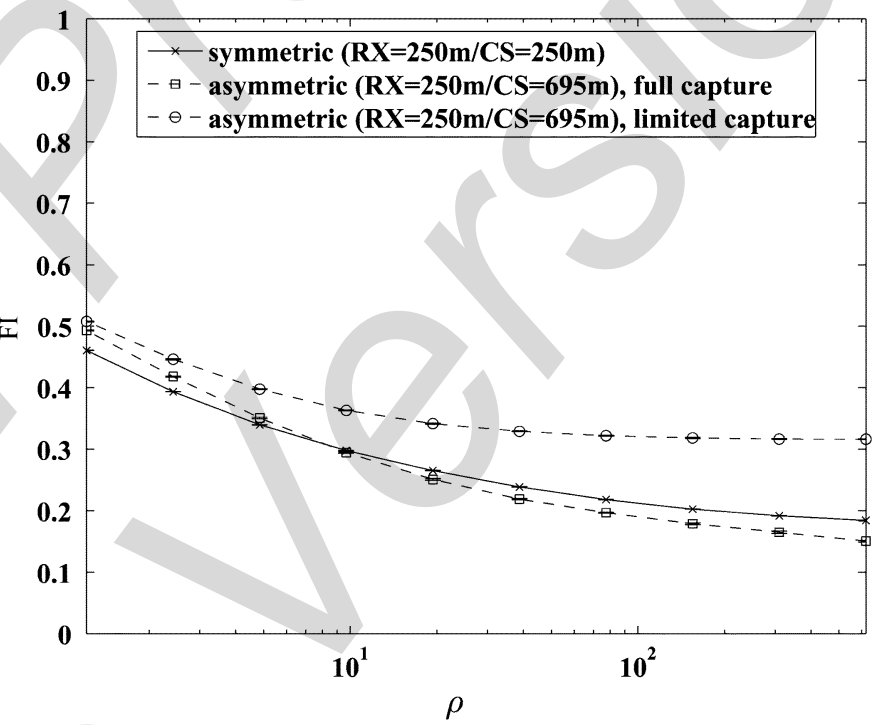

Fig. 15. Fairness index of the idealized protocol on the 2-D topology (simulation results).

The spatial reuse and fairness are reported in Fig. 14 and 15 respectively (the details of the simulation settings are described in Section III-C). Our study of the line topology showed that in the asymmetric case with limited capture, border effects can spread 5 hops away from the border; it was thus important to generate a sufficiently large topology to avoid that the performance of the protocol merely reflects the border effects. Now, a random topology has an inherent source of unfairness that is absent from regular topologies such as the line and is simply caused by the varying number of neighbors of each node. It explains why even at very low values of the access intensity $\rho$ the fairness index is not close to 1 , contrary to the line. Despite this "topological unfairness" (which would be present not only in CSMA/CA protocols but also in any access protocol that does not adapt explicitly to the varying node degree), we see that in 


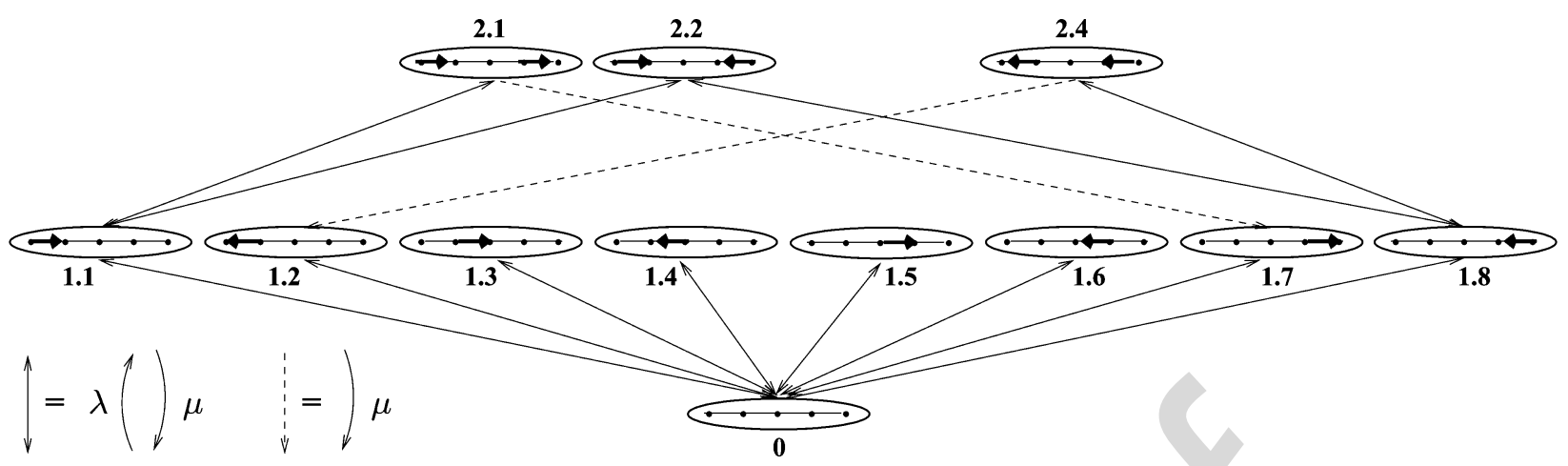

Fig. 16. The nonreversible Markov chain used to model the idealized protocol under asymmetric exclusion domains and limited capture.

this topology as well, the protocol with asymmetric exclusion domains and limited capture is the fairest when the value of $\rho$ becomes large.

\section{Markov Modeling}

The set of the valid transmission patterns (and thus the state space) is unchanged from the case with full capture effect. However, some states can now be reached only from a restricted set of lower states. Fig. 16 illustrates the Markov chain used to model the idealized protocol with limited capture effect. We see that, compared to the original Markov chain, two transition arrows have been removed: it accounts for the fact that the state at the top level can only be reached if the links become active in the right order.

Removing possible transitions between the states of the Markov chain breaks its regular structure, and the chain looses its reversibility property. This makes the analytical study of the chain very difficult as its stationary distribution does not have a nice product form anymore. However, a careful observation of the structure of the chain allows for the explanation of the results observed by simulation.

Let us first look back at the reversible case (corresponding to the two previous sections). For large values of $\rho$, the chain spends most of its time in the states of maximal spatial reuse. Indeed, when a transition occurs from a state at the top level to a state at the lower level (i.e., when an active link becomes idle), as $\lambda \gg \mu$, the next transition is most likely the reactivation of the same link, bringing the chain back to the same top level state. This explains why the protocol almost achieves maximal spatial reuse in the reversible case.

In the nonreversible case, this cannot happen anymore, because many of the transitions from top level states to lower level states cannot be reverted (see Fig. 16). Therefore, if the chain leaves a top level state, it might have to go down two or more levels before it can climb back to a top level state. The main consequence of this new dynamic is that the time spent in nonmaximal spatial reuse states (states below the top level) becomes nonnegligible.

In Proposition 1, we show that some states below the top level have a nontrivial (i.e., nonzero) stationary probability when $\rho \rightarrow$ $\infty$, contrary to the two previous cases. As a consequence, the average spatial reuse does no longer tend to the optimal value for increasing $\rho$.
Proposition 1: There exist transmission patterns of nonmaximal spatial reuse with a strictly positive stationary probability when $\rho \rightarrow \infty$.

Proof: Consider a transmission pattern $x^{*}$ of maximal spatial reuse, and another pattern $x^{\prime}$ obtained by removing an active link from $x^{*}$ and such that there is no possible transition (in the Markov chain) from $x^{\prime}$ to $x^{*}$ (which is only possible in the limited capture case). Assume furthermore that $x^{\prime}$ has $i$ active links. Now, the balance equation for $x^{\prime}$ reads

$$
\pi_{x^{\prime}} i \mu=\pi_{x^{*}} \mu+\sum_{y \in A_{x^{\prime}}} \pi_{y} \lambda
$$

where $A_{x^{\prime}}$ denotes the set of the states $y$ at level $i-1$ such that a transition from $y$ to $x^{\prime}$ is possible. Dividing both sides of this equation by $i \mu$, we obtain that $\pi_{x^{\prime}} \geq \pi_{x^{*}} / i$. Therefore, when $\rho$ tends to infinity, if $x^{*}$ has a non trivial asymptotic stationary probability, then so does $x^{\prime}$.

However, the loss in the spatial reuse is compensated by an increase in the fairness of the protocol. Indeed, contrary to the two previous cases, links that do not belong to a pattern of maximal spatial reuse do not necessarily get starved.

Proposition 2: There exist links that do not belong to the patterns of maximal spatial reuse but have a strictly positive probability of being active when $\rho \rightarrow \infty$.

Proof: Take a state $x^{\prime}$ as above. Remove one additional active link (next to the one removed to obtain $x^{\prime}$ from $x^{*}$ ) to obtain state $y$. From state $y$ it is now possible to reach a state $z$ that contains a link that does not belong to any patterns of maximal spatial reuse. We now show that this state has a nontrivial probability. Using the balance equations, we have

$$
\pi_{y}\left(\mu(i-1)+\lambda\left|A_{y}\right|\right)>\mu \pi_{z}+\mu \pi_{x^{\prime}}
$$

where $\left|A_{y}\right|$ is the number of states at level $i$ connected to $y$. As

$$
\pi_{z} i \mu>\lambda \pi_{y}>\frac{\lambda}{\mu(i-1)+\lambda\left|A_{y}\right|}\left(\mu \pi_{z}+\mu \pi_{x^{\prime}}\right)
$$

we obtain

$$
\pi_{z}\left(\mu i(i-1)+\lambda i\left|A_{y}\right|-\lambda\right)>\lambda \pi_{x^{\prime}}
$$


which implies that for $\rho \rightarrow \infty$

$$
\pi_{z}>\frac{1}{i\left|A_{y}\right|-1} \pi_{x^{\prime}}
$$

Therefore, if $x^{\prime}$ has a nontrivial asymptotic stationary probability, so does $z$, and hence there are links that do not belong to the patterns of maximal spatial reuse but have a strictly positive probability of being active when $\rho \rightarrow \infty$.

Proposition 2 implies that, on the line topology, the starvation effect can always be avoided. In addition, we can show that in the asymmetric case with limited capture links in opposite direction have the same probability of being active (this was not the case under full capture). Indeed given any transmission pattern, a link can become active if and only if there is no transmitting node in the carrier sensing range of its two end nodes. Thus, if link $j_{\text {left }}$ can become active so can link $j_{\text {right }}$.

Note that Propositions 1 and 2 are not restricted to the line topology and can be applied to any network topology provided that the corresponding Markov chain includes states of type $x^{*}$, $x^{\prime}, y$, and $z$.

\section{CONCLUSION}

In multihop ad hoc networks, CSMA/CA protocols can create a high level of unfairness among the network links. This unfairness appears even on the simplest regular network topologies where all nodes, except the ones at the border, have the same number of neighbors.

In this paper, we introduce a class of continuous Markov chain models which explains accurately this observation but also shows that the performance of these protocols vary with the capture and sensing capabilities of the network nodes.

On the positive side, we find that for a given (finite) access intensity $\rho, \mathrm{CSMA} / \mathrm{CA}$ protocols are fairer when the receiving and carrier sensing ranges are significantly different (asymmetric case) than when they have virtually the same values (symmetric case). On the negative side, we show that the price to pay for this higher fairness is a lower spatial reuse.

In addition, we demonstrate that, when $\rho \rightarrow \infty$, the capture capabilities of the network nodes play a decisive role on the performance of the protocol. In the asymmetric case with full capture (as well as in the commonly adopted symmetric case), the spatial reuse is maximal but all the links that do not belong to the patterns of maximal spatial reuse are starved. In contrast, in the asymmetric case with limited capture, the spatial reuse is not maximal but starvation can be avoided.

\section{APPENDIX}

In this Appendix, we establish the following lemma, which we use to prove Theorems 1 and 2 .

Lemma 1: Let $\{X(k), k \geq 1\}$, be an infinite sequence of discrete random variables defined by their distribution

$$
= \begin{cases}Z^{-1} r^{i}\left(\begin{array}{c}
k m-(m-1) i+n \\
i
\end{array}\right), & 0 \leq i \leq k \\
0, & \text { otherwise }\end{cases}
$$

where $m$ and $n$ are fixed finite integer parameters with $m \geq 2$ and $n \geq 0, r$ is a positive real number, and where

$$
Z=\sum_{i=0}^{k} r^{i}\left(\begin{array}{c}
k m-(m-1) i+n \\
i
\end{array}\right)
$$

is a normalizing constant. Then

$$
\lim _{k \rightarrow \infty} \frac{\mathbb{E}(X(k))}{k}=\frac{m r y_{1}{ }^{m-1}}{1+m r y_{1}{ }^{m-1}}
$$

where $y_{1}$ is the positive real root of $1-y-r y^{m}$.

Theorems 1 and 2 are two particular cases of this lemma, where the random variables $X(k)$ are the levels of the Markov chain (with $k=\lfloor(L+l-1) / l\rfloor$ ), and whose invariant distribution $N(i) \pi(i)$ coincides with the distribution $p_{k}(i)$ in the lemma, for particular values of $m, r$ and $n$ that differ for both theorems. As a result, the asymptotic average spatial reuse for large $L$ (and thus $k$ ) is given by (6), for the appropriate values of $m, r$ and $n$.

\section{A. Outline of the Proof of Lemma 1}

The proof can be subdivided in three main steps:

1) we first show that there is an integer $0<i_{k}^{*}<k$ such that $p_{k}\left(i_{k}^{*}\right)$ is maximal;

2) we then show that

$$
\lim _{k \rightarrow \infty} \frac{\mathbb{E}\left(X(k)-i_{k}^{*}\right)}{k}=0 .
$$

This implies that $\lim _{k \rightarrow \infty} \frac{\mathbb{E}(X(k))}{k}$ exists if and only if $\lim _{k \rightarrow \infty} \frac{i_{k}^{*}}{k}$ exists;

3) the last step of the proof consists in computing this limit, and showing that it is (6).

\section{B. Computation}

1) Step 1: We first show that the function $p_{k}(i)$ is maximum for some $0<i_{k}^{*}<k$, by using functions of the ratios of these quantities. We define the functions $\alpha_{k}(i)$ for $1 \leq i \leq k$ as

$$
\begin{aligned}
\alpha_{k}(i) & \doteq \frac{p_{k}(i)}{p_{k}(i-1)} \\
& =r \frac{\left(\begin{array}{c}
k m-(m-1) i+n \\
i
\end{array}\right)}{\left(\begin{array}{c}
k m-(m-1)(i-1)+n \\
i-1
\end{array}\right)} \\
& =\frac{r(k m-m(i-1)+n)_{m}}{i(k m-(m-1)(i-1)+n)_{m-1}}
\end{aligned}
$$

where the notation $(x)_{m}$ stands for $(x)_{m}=\prod_{j=0}^{m-1}(x-j)$. Therefore, the ratio between two consecutive terms is

$$
\begin{aligned}
\frac{\alpha_{k}(i+1)}{\alpha_{k}(i)}=\frac{i}{i+1} \cdot \frac{(k m-m i+n)_{m}}{(k m-m(i-1)+n)_{m}} \\
\frac{(k m-(m-1)(i-1)+n)_{m-1}}{(k m-(m-1) i+n)_{m-1}}
\end{aligned}
$$

$p_{k}(i) \doteq \mathbb{P}(X(k)=i)$ 
which can be rewritten as

$$
\begin{aligned}
& \frac{\alpha_{k}(i+1)}{\alpha_{k}(i)}=\frac{i}{i+1} \cdot \frac{k m-m i+n-m+1}{k m-m i+n+1} . \\
& \prod_{j=0}^{m-2} \frac{(k m-m i+n-j)(k m-(m-1)(i-1)+n-j)}{(k m-m(i-1)+n-j)(k m-(m-1) i+n-j)} .
\end{aligned}
$$

A close observation of each fraction allows to see that for $m>1$ each fraction is strictly smaller than one. Thus, $\alpha_{k}$ is a strictly decreasing function that ranges from $\alpha_{k}(1)=r((k-1) m+n+$ $1)>1$ to $\alpha_{k}(k)=r(m+n)_{m} /\left(k(k+m-1+n)_{m-1}\right)<1$. This implies that there is a value $0<i_{k}^{*}<k$ such that $\alpha_{k}\left(i_{k}^{*}+\right.$ $1) \leq 1$ and $\alpha_{k}\left(i_{k}^{*}\right)>1$. Consequently, $p_{k}\left(i_{k}^{*}\right)$ is maximal, and $\alpha_{k}\left(i_{k}^{*}+2\right)<1$.

2) Step 2: We now move on to the computation of the expected value of $X(k)$. We will see that the expected value is close to $i_{k}^{*}$ for large $k$; hence, we directly compute $\mathbb{E}\left(X(k)-i_{k}^{*}\right)$ instead of $\mathbb{E}(X(k))$. We also introduce the values $\hat{\imath}_{k}:=i_{k}^{*}+f(k)$ and $\check{\imath}_{k}:=i_{k}^{*}-f(k)$, where $f(k)$ is an integer function such that $\lim _{k \rightarrow \infty} f(k) / \sqrt{k}=0$ but $\lim _{k \rightarrow \infty} f(k) / \log k=\infty$, to decompose the summation in

$$
\mathbb{E}\left(X(k)-i_{k}^{*}\right)=\sum_{i=0}^{k}\left(i-i_{k}^{*}\right) p_{k}(i)
$$

in three terms

$$
\begin{aligned}
\mathbb{E}\left(X(k)-i_{k}^{*}\right) & =\sum_{i=0}^{\check{\iota}_{k}}\left(i-i_{k}^{*}\right) p_{k}(i) \\
& +\sum_{i=\check{\iota}_{k}+1}^{\hat{\imath}_{k}-1}\left(i-i_{k}^{*}\right) p_{k}(i)+\sum_{i=\hat{\imath}_{k}}^{k}\left(i-i_{k}^{*}\right) p_{k}(i)
\end{aligned}
$$

We show that each of these three terms is of order $o(k)$. For the first one, using the definition of $\alpha_{k}$ and the fact that it is a striclty decreasing function, we find that

$$
\begin{aligned}
0 \leq & \sum_{i=0}^{\check{c}_{k}}\left(i_{k}^{*}-i\right) p_{k}(i) \\
\leq & p_{k}\left(\check{\imath}_{k}\right) \sum_{i=0}^{\check{\imath}_{k}}\left(i_{k}^{*}-i\right) \\
\leq & p_{k}\left(\check{\imath}_{k}\right) \sum_{i=0}^{k}(k-i) \\
= & (k(k+1) / 2) p_{k}\left(\check{\imath}_{k}\right) \\
= & (k(k+1) / 2) p_{k}\left(i_{k}^{*}-1\right) \prod_{j=\check{c}_{k}+1}^{i_{k}^{*}-1}\left(\alpha_{k}(j)\right)^{-1} \\
= & (k(k+1) / 2) p_{k}\left(i_{k}^{*}-1\right) \\
& \quad \times \quad \prod_{j=0}\left(\alpha_{k}\left(i_{k}^{*}-1-j\right)\right)^{-1} \\
\leq & (k(k+1) / 2) p_{k}\left(i_{k}^{*}-1\right) \\
& \times\left(\alpha_{k}\left(i_{k}^{*}-1\right)\right)^{-f(k)+2} \\
\leq & (k(k+1) / 2)\left(\alpha_{k}\left(i_{k}^{*}-1\right)\right)^{-f(k)+2}
\end{aligned}
$$

where that last line follows from $p_{k}\left(i_{k}^{*}-1\right) \leq 1$. As $\alpha\left(i_{k}^{*}-1\right)>$ 1 , this expression tends to 0 when $k$ tends to infinity, which shows that the first term in $(8)$ is $o(k)$. The third term can be bounded in a similar manner, whereas the computation for the middle term reads

$$
\begin{aligned}
& \left|\sum_{i=\breve{\imath}_{k}+1}^{\hat{\imath}_{k}-1}\left(i-i_{k}^{*}\right) p_{k}(i)\right| \\
& \leq \sum_{i=\breve{\imath}_{k}+1}^{\hat{\imath}_{k}-1}\left|\left(i-i_{k}^{*}\right) p_{k}(i)\right| \\
& =\sum_{i=-f(k)+1}^{f(k)-1}|i| p_{k}\left(i+i_{k}^{*}\right) \\
& \leq \sum_{i=-f(k)}^{f(k)} f(k) p_{k}\left(i+i_{k}^{*}\right) \\
& \leq(2 f(k)+1) f(k) \\
& =o(k) .
\end{aligned}
$$

Combining the three terms, we conclude that (8) is of order $o(k)$, and thus that

$$
\lim _{k \rightarrow \infty} \frac{\mathbb{E}\left(X(k)-i_{k}^{*}\right)}{k}=0
$$

which implies in turn that $\lim _{k \rightarrow \infty} \mathbb{E}(X(k)) / k$ exists if and only if $\lim _{k \rightarrow \infty} i_{k}^{*} / k$ exists.

3) Step 3: The last step of the proof thus consists in computing the latter limit. We first extend the support of the function $\alpha_{k}(i)$ from $\mathbb{N}$ to $\mathbb{R}^{+}$, by observing in (7) that $\alpha_{k}(i)$ is well defined for noninteger values of $i$. The function $\alpha_{k}(x)$ over $x \in \mathbb{R}^{+}$, is a continuous, strictly decreasing function, and we can therefore find the value $x \in \mathbb{R}$ such that $\alpha_{k}(x)=1$. Clearly, $\left|x-i_{k}^{*}\right|<1$, so that $\lim _{k \rightarrow \infty} x / k=\lim _{k \rightarrow \infty} i_{k}^{*} / k$. We need therefore to compute the root $x \in \mathbb{R}^{+}$of the equation $\alpha_{k}(x)=1$, which can be expanded from (7) as

$$
\frac{r \prod_{j=0}^{m-1}(k m-m(x-1)+n-j)}{x \prod_{j=0}^{m-2}(k m-(m-1)(x-1)+n-j)}=1 .
$$

Dividing the numerator and denominator by $k^{m}$, we obtain

$$
\begin{aligned}
r \prod_{j=0}^{m-1}(m- & \left.m\left(\frac{x}{k}-\frac{1}{k}\right)+\frac{n-j}{k}\right) \\
& =\frac{x}{k} \prod_{j=0}^{m-2}\left(m-(m-1)\left(\frac{x}{k}-\frac{1}{k}\right)+\frac{n-j}{k}\right)
\end{aligned}
$$

Taking the limit of both sides for $k \rightarrow \infty$ we get

$$
r \prod_{j=0}^{m-1}\left(m-m \frac{x}{k}\right)=\frac{x}{k} \prod_{j=0}^{m-2}\left(m-(m-1) \frac{x}{k}\right),
$$

and solving for $r$, we obtain

$$
r=\frac{x}{m(k-x)}\left(1+\frac{x}{m(k-x)}\right)^{m-1} .
$$


Let

$$
y=\left(\frac{x}{m(k-x) r}\right)^{1 /(m-1)}
$$

or equivalently

$$
\frac{x}{k}=\frac{m r y^{m-1}}{1+m r y^{m-1}} .
$$

Using this change of variables in (9), we get

$$
r=r y^{m-1}\left(1+r y^{m-1}\right)^{m-1}
$$

which we can recast as

$$
1-y-r y^{m}=0 .
$$

By Descartes' rule of signs, the difference between the number of positive real roots of a real polynomial and the number of changes of signs of the sequence of its coefficients is always an even, non positive number. The polynomial $1-y-r y^{m}$ has one change of sign, and therefore only one positive real root (as we could expect since $\alpha(x)$ is continuous and strictly decreasing for $x \in \mathbb{R}^{+}$), which we denote by $y_{1}$.

Because of (10), we have therefore proven that

$$
\lim _{k \rightarrow \infty} \frac{\mathbb{E}(X(k))}{k}=\frac{m r y_{1}{ }^{m-1}}{1+m r y_{1}{ }^{m-1}}
$$

where $y_{1}$ is the real solution of (11).

\section{REFERENCES}

[1] IEEE802.11, Part 11: Wireless LAN Medium Access Control (MAC) and Physical Layer (PHY) Specifications Aug. 1999, IEEE Std..

[2] X. Wang and K. Kar, "Throughput modelling and fairness issues in CSMA/CA based ad-hoc networks," in Proc. INFOCOM, Miami, FL, 2005.

[3] M. Durvy and P. Thiran, "A packing approach to compare slotted and nonslotted medium access control," in Proc. INFOCOM, Barcelona, Spain, 2006.

[4] M. M. Carvalho and J. J. Garcia-Luna-Aceves, "A scalable model for channel access protocols in multihop ad hoc networks," in Proc. MobiCom, 2004, pp. 330-344, ACM Press.

[5] M. Garetto, T. Salonidis, and E. Knightly, "Modeling per-flow throughput and capturing starvation in CSMA multihop wireless networks," in INFOCOM, Barcelona, Spain, 2006.

[6] K. Medepalli and F. Tobagi, "Towards performance modeling of IEEE 802.11 based wireless networks: A unified framework and its applications," in Proc. INFOCOM, Barcelona, Spain, 2006.

[7] M. Garey and D. Johnson, Computers and Intractability: A Guide to the Theory of NP-Completeness. New York: Freeman, 1983.

[8] L. Tassiulas and A. Ephremides, "Stability properties of constrained queueing systems and scheduling for maximum throughput in multihop radio networks," IEEE Trans. Autom. Control, vol. 37, no. 12, pp. 1936-1949, 1992.

[9] E. Modiano, D. Shah, and G. Zussman, "Maximizing throughput in wireless networks via gossip," in Proc. ACM SIGMETRIC/Perform., 2006.

[10] K. Jung and D. Shah, "Low delay scheduling algorithms," in Proc. IEEE ISIT, 2007.

[11] G. Bianchi, "Performance analysis of the IEEE 802.11 distributed coordination function," IEEE J. Sel. Areas Commun., vol. 18, no. 3, 2000.

[12] F. Calì, M. Conti, and E. Gregori, "IEEE 802.11 protocol: Design and performance evaluation of an adaptive backoff mechanism," IEEE J. Sel. Areas Commun., vol. 18, no. 9, pp. 1774-1780, 2000.
[13] F. Calì, M. Conti, and E. Gregori, "Dynamic tuning of the IEEE 802.11 protocol to achieve a theoretical throughput limit," IEEE/ACM Trans. Netw., vol. 8, no. 6, pp. 785-799, 2000.

[14] M. Zorzi and R. Rao, "Capture and retransmission control in mobile radio," IEEE J. Sel. Areas Commun., vol. 12, no. 8, pp. 1289-1298, Oct. 1994.

[15] C. T. Lau and C. Leung, "Capture models for model packet radio networks," IEEE Trans. Commun., vol. 40, no. 5, pp. 917-925, May 1992.

[16] W. Luo and A. Ephremides, "Power levels and packet lengths in random multiple access," IEEE Trans. Inf. Theory, vol. 48, no. 1, pp. 46-58, Jan. 2002.

[17] Z. Hadzi-Velkov and B. Spasenovski, "On the capacity of IEEE 802.11 DCF with capture in multipath-faded channels," Int. J. Wireless Inf. Net., vol. 9, no. 3, pp. 191-199, July 2002.

[18] Z. Hadzi-Velkov and B. Spasenovski, "Capture effect with diversity in IEEE 802.11b DCF," in Proc. IEEE ISCC'03, Jun. 2003.

[19] H. S. Chhaya and S. Gupta, "Performance modeling of asynchronous data transfer methods of IEEE 802.11 mac protocol," Wirel. Netw., vol. 3, no. 3, pp. 217-234, 1997.

[20] N. Gupta and P. R. Kumar, "A performance analysis of the IEEE 802.11 wireless LAN medium access control," Commun. Inf. Syst., vol. 3, no. 4, pp. 279-304, 2003.

[21] Y. Wang and J. J. Garcia-Luna-Aceves, "Modeling of collision avoidance protocols in single-channel multihop wireless networks," Wirel. Netw., vol. 10, no. 5, pp. 495-506, 2004.

[22] F. A. Tobagi, "Modeling and performance analysis of multihop packet radio networks," Proc. IEEE, vol. 75, no. 1, pp. 135-155, 1987.

[23] R. Boorstyn, A. Kershenbaum, B. Maglaris, and V. Sahin, "Throughput analysis in multihop CSMA packet radio networks," IEEE Trans. Commun., vol. 35, no. 3, pp. 267-274, 1987.

[24] A. Proutiere, Y. Yi, and M. Chiang, "Throughput of random medium access control: An asymptotic approach," in Proc. CISS'08, Mar. 2008.

[25] A. Jindal and K. Psounis, "Achievable rate region and optimality of multihop wireless 802.11-scheduled networks," in Proc. ITA'08, Jan. 2008.

[26] C. Bordenave, D. McDonald, and A. Proutiere, "Performance of random medium access control: An asymptotic approach," in Proc. ACM Sigmetrics'08, June 2008.

[27] M. Hira, F. Tobagi, and K. Medepalli, "Throughput analysis of a path in an IEEE 802.11 multihop wireless network," in Proc. IEEE Wireless Commun. Netw. Conf. (WCNC 2007), Mar. 2007, pp. 441-446.

[28] L. T. Nguyen, R. Beuran, and Y. Shinoda, "Performance analysis of IEEE 802.11 in multihop wireless networks," Mobile Ad-Hoc and Sensor Networks, ser. Lecture Notes in Computer Science, vol. 4864, pp. 326-337, 2007.

[29] P. C. Ng and S. C. Liew, "Throughput analysis of IEEE 802.11 multihop ad hoc networks," IEEE/ACM Trans. Netw., vol. 15, no. 2, pp. 309-322, 2007.

[30] M. Durvy, O. Dousse, and P. Thiran, "Modeling the 802.11 protocol under different capture and sensing capabilities," in INFOCOM (Minisymposium), Anchorage, AK, 2007.

[31] M. Durvy and P. Thiran, "Understanding the gap between the IEEE 802.11 protocol performance and the theoretical limits," in Proc. SECON, Reston, VA, 2006.

[32] V. Bharghavan, A. Demers, S. Shenker, and L. Zhang, "MACAW: A media access protocol for wireless LAN's," in Proc. SIGCOMM, 1994, pp. 212-225, ACM Press.

[33] R. Jain, The Art of Computer Systems Performance Analysis. New York: Wiley, 1991.

[34] Discrete Event Simulator for the Idealized 802.11 Protocol, [Online] Available: http://icapeople.epfl.ch/mdurvy/research.html

[35] F. P. Kelly, "Loss networks," Ann. Appl. Probab., vol. 1, no. 3, pp. 319-378, 1991.

[36] D. Y. Burman, J. P. Lehoczky, and Y. Lim, "Insensitivity of blocking probabilities in a circuit-switching network," J. Appl. Probab., vol. 21 , no. 4, pp. 850-859, 1984.

[37] Y. Baryshnikov, E. G. Coffman Jr., and P. Jelenković, "Space filling and depletion," J. Appl. Probab., vol. 41, no. 3, pp. 691-702, 2004.

[38] A. Kochut, A. Vasan, A. Shankar, and A. Agrawala, "Sniffing out the correct physical layer capture model in 802.11b," in Proc. ICNP, 2004.

Mathilde Durvy (S'02) received the M.Sc. degree in 2002 and the Ph.D. degree in 2007 in communication systems from EPFL, Lausanne, Switzerland.

Her research interests are in the design and analysis of medium access control protocols for multihop ad hoc networks.

In 2005, she received an Infocom travel grant and in 2007, she was a finalist of the Google Anita Borg scholarship. 
Olivier Dousse (M'00) received the M.Sc. degree in physics from the Swiss Federal Institute of Technology at Lausanne, Switzerland (EPFL) in 2000, and the $\mathrm{Ph} . \mathrm{D}$. degree in communication systems from the same institution in 2005.

From 2006 to 2008, he was Research Scientist at Deutsche Telekom Laboratories in Berlin. He is currently a member of research staff at Nokia Research Center in Lausanne. His research interests are in stochastic models for communication networks.

Dr. Dousse received the honorable mention of the 2005 ACM Doctoral Dissertation Competition, and he was awarded the Top 3 Student Prize of the EPFL Graduate School in communication systems in 2001. He was runner-up for the IEEE-INFOCOM Best Paper Award in 2003.

Patrick Thiran (S'89-M'97) received the electrical engineering degree from the Université Catholique de Louvain, Louvain-la-Neuve, Belgium, in 1989, the M.S. degree in electrical engineering from the University of California at Berkeley, USA, in 1990, and the Ph.D. degree from EPFL, in 1996.

$\mathrm{He}$ is an Associate Professor at EPFL. He became an Adjunct Professor in 1998, an Assistant Professor in 2002 and an Associate Professor in 2006. From 2000 to 2001, he was with Sprint Advanced Technology Labs, Burlingame, CA. His research interests include communication networks, performance analysis, dynamical systems, and stochastic models. He is currently active in the analysis and design of wireless multihop networks and in network monitoring.

Dr. Thiran served as an Associate Editor for the IEEE TRANSACTIONS ON CIRCUITS AND SYSTEMS in 1997-1999, and he is currently an Associate Editor for the IEEE/ACM TRANSACTIONS ON NETWORKING. He was the recipient of the 1996 EPFL Ph.D. award. 


\title{
Self-Organization Properties of CSMA/CA Systems and Their Consequences on Fairness
}

\author{
Mathilde Durvy, Student Member, IEEE, Olivier Dousse, Member, IEEE, and Patrick Thiran, Member, IEEE
}

\begin{abstract}
Decentralized medium access control schemes for wireless networks based on CSMA/CA, such as the IEEE 802.11 protocol, are known to be unfair. In multihop networks, they can even favor some links to such an extent that the others suffer from virtually complete starvation. This observation has been reported in quite a few works, but the factors causing it are still not well understood. We find that the capture effect and the relative values of the receive and carrier sensing ranges play a crucial role in the performance of these protocols. Using a simple Markovian model, we show that an idealized CSMA/CA protocol suffers from starvation when the receiving and sensing ranges are equal, but quite surprisingly that this unfairness is reduced or even disappears when these two ranges are sufficiently different. We also show that starvation has a positive counterpart, namely organization. When its access intensity is large the protocol organizes the transmissions in space in such a way that it maximizes the number of concurrent successful transmissions. We obtain exact formulæ for the so-called spatial reuse of the protocol on large line networks.
\end{abstract}

Index Terms-Ad hoc networks, CSMA/CA, medium-access control (MAC), modeling, multihop, performance analysis.

\section{INTRODUCTION}

$\mathbf{T}$ HE IEEE 802.11 [1] medium-access control (MAC) protocol is unfair. This statement has been so often repeated that it is now widely accepted. Indeed, in single-hop networks where all nodes can communicate directly, it has been shown that the Binary Exponential Backoff (BEB) of the 802.11 protocol creates situations where a single node has an almost exclusive access to the communication channel. In multihop networks, similar starvation phenomena have been reported ([2]-[6]). However, contrary to the single-hop case, very few models are able to explain the starvation problems encountered in multihop topologies. Existing models often concentrate on very small network topologies or describe how a specific feature of the 802.11 protocol affects its fairness. In addition, it is common to assume that the receiving and carrier sensing ranges are equal, or to neglect the the so-called "capture effect" and to assume instead that two packets received at the same

Manuscript received December 05, 2007; revised November 19, 2008. Current version published February 13, 2009. The work was supported in part by the National Competence Center in Research on Mobile Information and Communication Systems (NCCR-MICS), a center supported by the Swiss National Science Foundation under Grant 5005-67322.

M. Durvy and P. Thiran are with EPFL, CH-1015 Lausanne, Switzerland (e-mail: mathilde.durvy@epfl.ch; patrick.thiran@epfl.ch).

O. Dousse is with Nokia Research Center, CH-1015 Lausanne, Switzerland (e-mail: olivier.dousse@nokia.com).

Communicated by E. Modiano, Associate Editor for Communication Networks.

Digital Object Identifier 10.1109/TIT.2008.2011427 time always collide. We elaborate on existing models and their assumptions in Section II.

The goal of this work is to shed some light on how such assumptions affect the performance of general CSMA/CA protocols. Our approach is the following. Instead of concentrating on specific implementation aspects, we consider an idealized protocol that retains the key features of CSMA/CA protocols such as their carrier sensing, collision avoidance, and backoff mechanisms. Section III describes this protocol and the metrics used to evaluate its performance. We use continuous time Markov chains to describe the dynamics of the protocol. We find that even though this idealized protocol does not suffer from the well-known problems that have already been identified in single-hop and multihop networks, it is still subject to the starvation phenomenon described in the literature. However, we observe that the starvation phenomenon results from an optimal organization of the transmissions in space. Indeed, at high access intensities the idealized protocol maximizes, in a completely decentralized way, the number of simultaneous successful transmissions in the network. This is quite surprising as the problem of finding the transmission patterns that maximize spatial reuse in a general network is NP-Complete (by equivalence with the maximal independent set problem [7]). Following the work of [8], [9] and [10] have recently proposed several distributed algorithms to approach this goal ${ }^{1}$. Yet, we also show that the performance of the protocol are very sensitive to the assumptions made in terms of carrier sensing range and capture effect.

The main contribution of this paper is the explanation of the starvation and the organization phenomenons. In particular, we show under which condition these phenomenons occur and why. We separate our analysis into three classes of assumptions, each leading to a different Markov chain structure. Section IV covers the simplest case, that has previously been studied in the literature. It includes known results together with our own results. The purpose of this section is to keep the paper self-contained and to serve as a reference case for the study in the two next sections.

In Sections V and VI, we develop new Markov models to address the cases where the nodes' receiving range and carrier sensing range are significantly different. These two sections differ in the underlying assumptions on the nodes' capture capability, and consequently in the structure of the Markov models involved. In particular, in terms of their reversibility.

Finally, the Appendix contains a lemma used to derive exact formulæ for the performance of the idealized protocol under a simple line network topology.

\footnotetext{
${ }^{1}$ In fact, they consider a slightly more general problem, the maximal weight independent set problem.
} 


\section{RELATED WORK}

For a long time, models for the 802.11 protocol have been limited to the single-hop setting and numerous works have extended the original papers of Bianchi [11] and Cali, Conti, and Gregori [12], [13]. Similarly, the capture effect has first been studied for slotted Aloha schemes [14]-[16], and more recently for single-hop 802.11 networks [17], [18]. Unfortunately, models for the 802.11 protocol in the single-hop setting are not easy to extend to the multihop setting, because they rely explicitly on the central assumption that all nodes can hear each other and that there are no hidden terminals. Lately however, several papers have attempted to model the behavior of CSMA/CA protocols in the multihop setting. This section is dedicated to such papers, as our work is devoted to CSMA/CA multihop networks. In particular, we concentrate on their ability to characterize the spatial reuse/fairness performance of these protocols. We also try to highlight the assumptions made in terms of carrier sensing and capture models.

[19] makes a first step towards a multihop analysis of the 802.11 protocol. Their model accounts for the capture effect but is valid only for a limited number of hidden terminals. [20] and [21] are really the first to consider the operation of the 802.11 protocol on a real multihop network. Their models either perform a fixed point iteration or use numerical methods to obtain the throughput performance of the 802.11 protocol on specific network topologies (a ring for [20] and a Poisson topology for [21]). The work of [2] forms the basis our paper. It extends the Markov chain formalism used by [22], [23] to model the CSMA protocol to an idealized CSMA/CA protocol. The strength of this model is to preserve the dependence between nodes, which is typical of CSMA and CSMA/CA protocols. Using this model, [2] is probably the first to predict and explain the starvation phenomenon on general multihop topologies. The ability of CSMA/CA protocols to reach a high spatial reuse or throughput when all nodes of a multihop networks are saturated has been highlighted in [3], [24], and [25]. [26] studies the throughput region using mean-field techniques, which are valid in the limit when the number of nodes within the same interference range is arbitrary large but allow to include adaptive back-offs. However, all these works ([20], [21], [3], [2], [24]-[26]) consider only the case where the receiving and carrier sensing ranges are equal, and do not take the capture effect into account.

[4]-[6], [27], [28], and [29] relax the assumption of equal receiving and carrier sensing ranges but still neglect the capture effect. These papers present results in terms of throughput and fairness, but only the models of [5] and [6] are able to predict the starvation phenomenon. The work of [6] does not investigate the effect of the carrier sensing range on the performance of the 802.11 protocol, it rather concentrates on the role played by the minimum contention window in the starvation phenomenon. [27], [28], and [29] concentrate on the end-to-end throughput of a single flow or path. [5] uses a 50 node 2-D topology on a $1000 \times 1000 \mathrm{~m}$ square area to validate its model. The receiving range is set to $200 \mathrm{~m}$ and the carrier sensing range to either 200 or $400 \mathrm{~m}$. [5] observes that the unfairness of the 802.11 protocol

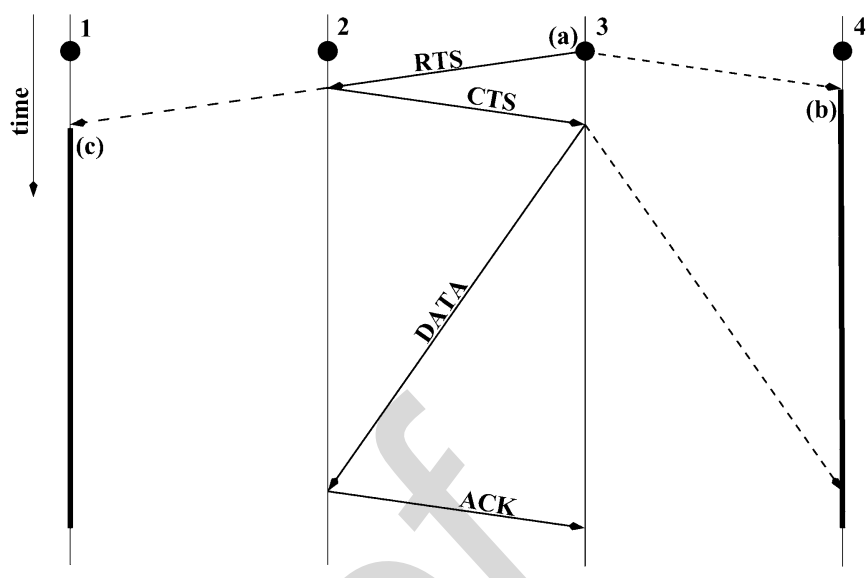

Fig. 1. A typical exchange between Node 3 and Node 2. At point (a) the backoff timer of Node 3 reaches zero, Node 3 sends a RTS packet to Node 2. Upon receiving the RTS (b) (respectively CTS (c)) Node 4 (respectively Node 1) sets its NAV (bold line) to cover the duration of the exchange between Node 3 and Node 2.

is higher at a large carrier sensing range. Although the model of [4] does not fully predict the starvation phenomenon, the authors also observe it by simulation. Interestingly, they use the same experimental setting as [5] (with different node positions), but their simulations show that the unfairness of the 802.11 protocol is now higher at a small carrier sensing range. These apparently contradicting results are probably a consequence of the limited amount of multihopping possible in the topology used in [5] and [4] (indeed, the receiving and carrier sensing ranges are such that they cover a large part of the network).

In this paper, we recognize the need to consider larger network topologies with reduced border effects to obtain more predictable results. We extend the work of [2] to 1) allow for different receive and carrier sensing ranges, 2) account for the capture effect, and 3) provide closed-form expressions for the spatial reuse of CSMA/CA protocols on large line networks. We favor the model of [2] over the other models as it makes the starvation phenomenon directly apparent in its equations.

Part of this work has been presented in the conference papers [3] and [30].

\section{FRAMEWORK}

\section{A. Medium-Access Control (MAC) Layer}

1) Protocol Overview: We first provide a high-level overview of the CSMA/CA protocol that we analyze.

In this protocol, a node intending to transmit senses first the medium. Physical and virtual carrier sensing mechanisms are used to determine the state of the medium. The physical carrier sensing is provided by the physical layer. The virtual carrier sensing is done through an RTS/CTS handshake, as illustrated in Fig. 1: The sender first sends a "request to send" packet (RTS), to which the receiver answers with a "clear to send" packet (CTS). Both packets contain information about the time at which the exchange will be completed, so that overhearing nodes can update their network allocation vector (NAV) and refrain from emitting during the exchange. In this way, interferences from other transmissions are avoided. 


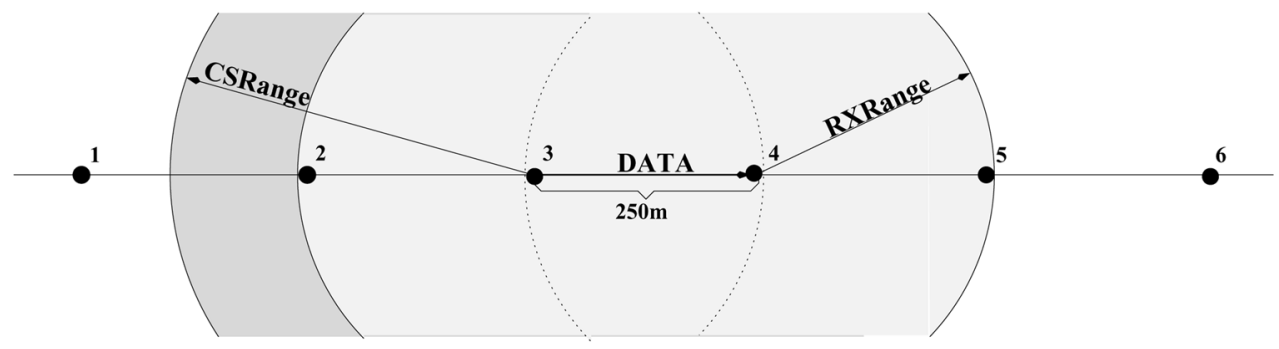

Fig. 2. All links with an end-node in the gray domain around the active link are silenced (nine directed links are thus silenced). As the nodes in the transmitter's CSRange are in its RXRange, the exclusion domain is symmetric.



Fig. 3. As the CSRange is much larger than the RXRange, more nodes are silenced around the transmitter (Node 3). The exclusion domain is asymmetric.

However, this mechanism has the drawback of synchronizing transmission attempts: If several nodes have been waiting to transmit due to an ongoing transmission, they will attempt to access the channel as soon as the transmission completes (i.e., as soon as they sense no activity on the channel). This creates predictable collisions. To avoid this problem, each node maintains a backoff timer, which is initialized to a random value chosen according to some backoff distribution. Timers run when the channel is idle; when a node senses the channel busy, it temporarily freezes its timer. Nodes may start emitting only when their timer reaches zero. After each transmission, the emitter resets its timer to a new random value. This mechanism causes random delays between the end of a transmission and the start of the next one, and spreads transmission attempts in time.

2) Assumptions: In this paper, we consider an idealized CSMA/CA protocol in order to capture the essential features of CSMA/CA systems, and leave aside the effects due to the imperfection of real protocols (we refer the reader to [31] for an overview of those effects in IEEE 802.11).

First, we assume that the RTS/CTS handshake is so short that its duration can be ignored in the model. In addition, we consider a continuous backoff distribution, instead of the discrete distribution implemented in actual protocols, so that two timers have a zero probability to expire at the same time. These two assumptions imply that no collision occurs due to simultaneous transmission attempts.

We also assume that nodes maintain a separate backoff timer for each of their outgoing links, as recommended by [32] in an attempt to guarantee per link fairness at least in the context of single-hop networks.

3) Interaction With the Physical Layer: We model the physical layer using three parameters: the receiving range, the carrier sensing range and the interference range.

- The receiving range (RXRange) is the maximum distance from the source at which a packet can be successfully re- ceived in the absence of interfering nodes. In practice, the RXRange depends on the rate at which a packet is sent. In our model, we assume that all packets (including RTS and CTS packets) are sent with the same rate, so that the same ranges apply to any transmission. We say that there is a link from the source to each of the nodes in its RXRange.

- The carrier sensing range (CSRange) is the maximum distance from the emitter at which its transmission can still be detected, but not necessarily decoded. In this way, all nodes within the CSRange of an emitter sense the channel busy during its transmission. The CSRange is always larger than or equal to the RXRange.

After the initial RTS-CTS handshake, nodes in the RXRanges of the sender and the receiver are silenced by their virtual carrier sensing mechanism. In addition, nodes within CSRange of the sender are kept from sending by their physical carrier sensing mechanism. We refer to the domain silenced around an active link by its virtual and physical carrier sensing as its exclusion domain. In this work we distinguish between symmetric exclusion domains (Fig. 2, Section IV) and asymmetric exclusion domains (Fig. 3, Sections V and VI).

- The interference range (IRange) is the maximum distance from the receiver at which an interferer can prevent the successful decoding of a transmission. Unlike the other two ranges, the interference range follows from a SINR constraint at the receiver and depends on the actual distance between the emitter and the receiver. The IRange is the largest when this distance is equal to the RXRange, and we denote this maximal interference range by IRange*.

4) Collisions: A collision is the failure of a packet transmission due to the interference from another concurrent transmission. In our framework, collisions are avoided if the protocol successfully prevents all nodes in the interference range of the 
receiver to start a transmission. In the sequel, we will assume that it is the case. In other words, we assume that any node in the interference range of a receiver is also in the exclusion domain of the receiver's link. This happens if

$$
\text { RXRange } \geq \text { IRange* }
$$

or if

$$
\text { CSRange } \geq \text { IRange }^{*}+\text { RXRange }
$$

or of course if no node happens to lie in the interference range of the receiver (for example because of a regular placement of the nodes).

5) Capture Effect: However, the above assumption does not completely prevent overlapping transmissions: Assume that a node in the receiver's CSRange but outside of the link's exclusion domain (for example Node 6 in Fig. 3) starts to transmit. As the two nodes (Node 4 and Node 6 in the example) are within CSRange of each other, the new transmission (from Node 6) can be sensed at the receiver (Node 4) and thus interferes with the ongoing transmission (from Node 3 to 4 ). However, because of the assumption (1), the new transmitter (Node 6) is necessarily outside the receiver's IRange, so that the interference is not strong enough to create a collision. The signal-to-interference ratio at the receiver (Node 4) is still high enough for successful decoding the ongoing transmission and the new interfering signal is simply ignored: this is called the capture effect. In this work, we will consider two different models for capture effects, described, respectively, in Sections V-A and VI-A.

\section{B. Metrics}

Denote by $L$ the number of node pairs that can communicate (i.e., are in receiving range of each other). There are thus $2 L$ directed links in the network. We use two metrics to characterize the performance of our protocol.

1) Average Spatial Reuse $(\sigma)$ : We define the average spatial reuse as the number of active links normalized by $L$ (and averaged over time) in the network.

2) Fairness Index (FI): To assess the MAC layer fairness of the protocol we use Jain's Fairness Index [33]. Denote by $p(j)$ the probability that link $j$ be active under a given medium access control protocol. The link fairness index of the protocol is

$$
\mathrm{FI}=\frac{\left(\sum_{j} p(j)\right)^{2}}{2 L \sum_{j} p(j)^{2}} .
$$

The maximum fairness index is 1 . It corresponds to a network where all links access the channel equally. Yet, if only $k$ of the directed links have an equal access to the channel and the remaining links have no access to the channel, the fairness index is $k /(2 L)$.

\section{Problem Setting}

Our analysis assumes saturated traffic conditions (i.e., nodes always have a packet to send to each of their one-hop neighbors) and, unless otherwise specified, we consider exponentially distributed backoff and exchange times.

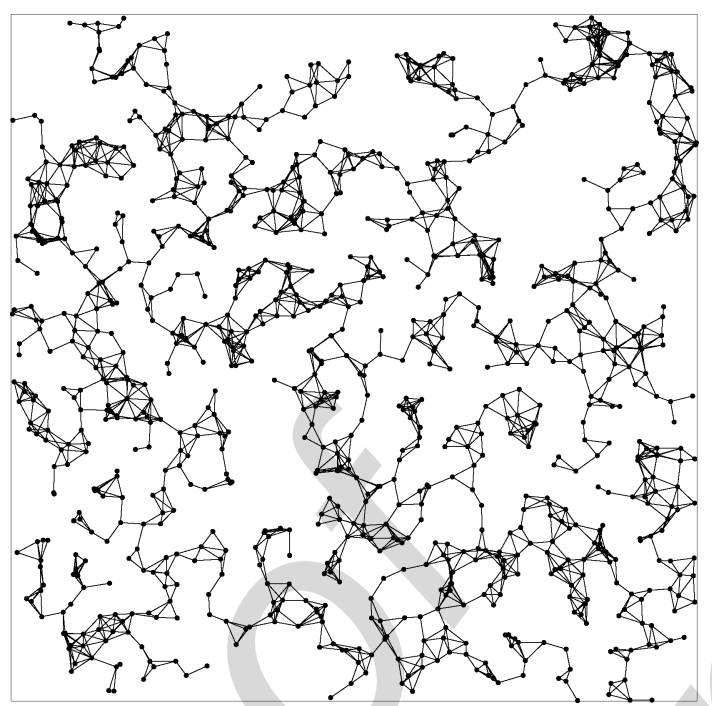

Fig. 4. Random 2-D topology.

All simulations in this paper were performed using a dedicated event driven simulator for our idealized protocol (code available at [34]). We simulated two different network topologies: a line network topology (50 equally spaced nodes at a distance of $250 \mathrm{~m}$ ) and a random 2-D topology (1065 nodes are randomly deployed on a $6500 \times 6500$ area, the isolated nodes are then removed to keep a connected component of 970 nodes, the average node degree is approximately 5 (Fig. 4)). In both topologies, the RXRange is $250 \mathrm{~m}$. For symmetric exclusion domains, the IRange* and the CSRange are equal to $250 \mathrm{~m}$ as well. For asymmetric exclusion domains, the IRange* is $445 \mathrm{~m}$ and the CSRange is equal to $550 \mathrm{~m}$ for the line and to $695 \mathrm{~m}$ for the random 2-D topology (this is necessary to make the protocol collision free). The simulations correspond to approximately 15 $\mathrm{min}$ of traffic. To provide accurate results, each simulation is repeated 20 times (using different random seeds). The presented results consist of the values averaged over the 20 experiments and, of the $95 \%$ confidence intervals.

\section{THE SyMmetric CASE}

\section{A. Specific Assumptions}

The exclusion domain around a link is symmetric if the set of nodes silenced by it is the same as the set silenced by the reverse link (the link with permuted emitter and receiver). In our physical layer model, this happens if no node is located outside the RXRange of the transmitter while being located inside its CSRange.

Exclusion domains are always symmetric if the CSRange and the RXRange are equal. However, symmetric exclusion domains often arise when the network topology is regular (see example in Fig. 2). For example, indoor environments with their carefully designed network topologies and strong signal attenuation may lead to such a situation.

The case with symmetric exclusion domain has already been studied in [2] using a Markov model very similar to the one presented below, which is a class of loss networks [35]. This section 


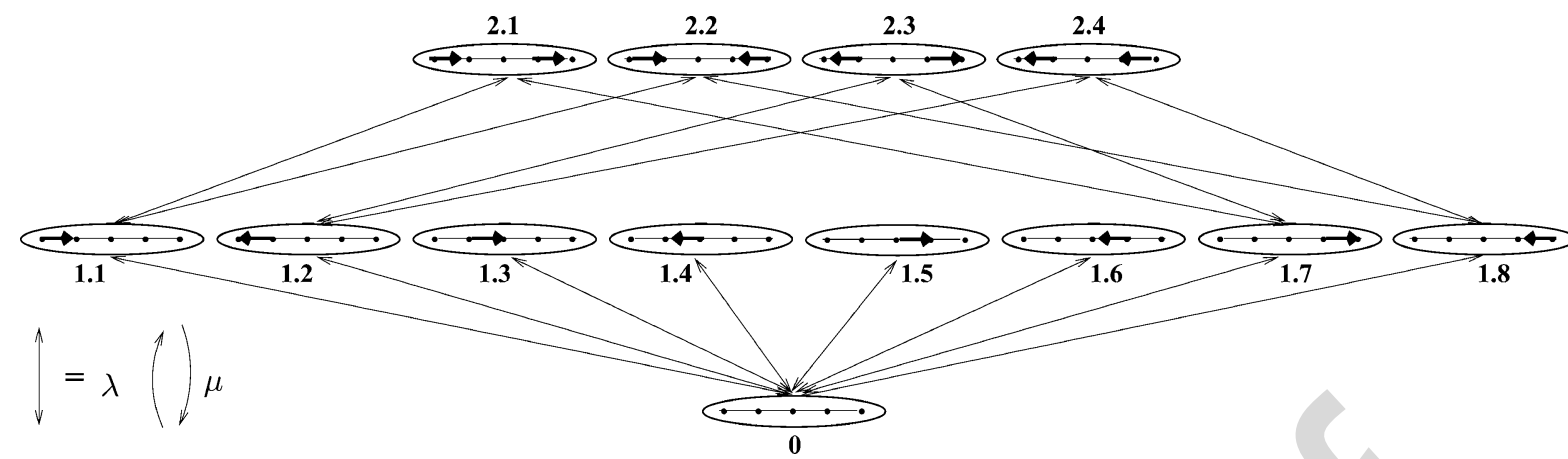

level 2

$N(2)=4$ no active link, $N(1)=8$ states with 1 active link, and $N(2)=4$ states with 2 active links.

extends the results of [2] by looking at a specific topology: the line topology. In particular, we obtain a closed-form formula for the spatial reuse of the protocol on large line networks (Theorem $1)$.

\section{B. Markov Modeling}

The dynamics of our idealized protocol can be described by a continuous Markov chain. At any given time, a set of directional links is active in the network. Such a set is called a transmission pattern and defines a state of the Markov chain. Clearly, only patterns that respect the constraints set by the exclusion domains of active links are possible. Hence the set of all valid patterns forms the state space of the Markov chain. We say that a state is at level $i$ if the corresponding transmission pattern has exactly $i$ active links, and we denote by $N(i)$ the number of such states. The states at the highest level are called patterns of maximal spatial reuse: they will be of particular interest for the analysis of the Markov chain.

Transitions in the Markov chain can only occur between states that are separated by one level: A transition between a state at level $i$ and a state at level $i-1$ corresponds to the completion of a transmission, and a transition between a state at level $i$ and a state at level $i+1$ corresponds to the beginning of a new transmission (which is of course possible only if the new link does not lie in the exclusion domain of already active links).

Denote by $\mu^{-1}$ the average exchange time and by $\lambda^{-1}$ the average backoff time. The transition rate between a state at level $i$ and a state at level $i-1$ (respectively, at level $i+1$ ) is $\mu$ (resp. $\lambda$ ). Fig. 5 gives an example of such a Markov chain. Finally, we define the access intensity as $\rho:=\lambda / \mu$.

The stationary distribution of this reversible Markov chain can be computed using the global balance equations and has a product form. Indeed, the stationary probability of any state at level $i$ is equal to

$$
\pi(i)=\rho^{i} \pi(0)
$$

where $\pi(0)$ is the stationary probability of the empty transmission pattern which is equal to

$$
\pi(0)=\left(\sum_{i} N(i) \rho^{i}\right)^{-1} .
$$

This form of stationary distributions first appeared in the context of circuit-switched loss networks [35] and it is known [36] that (2) and (3) are insensitive to the exchange time distribution. The average spatial reuse is then

$$
\sigma=\frac{1}{L} \sum_{i} i N(i) \pi(i)
$$

where $L$ is the number of undirected links in the network. To derive the long-term fairness of the protocol, we also need to compute the probability that a specific link is active. A link $j$ is active if the chain is in a state whose transmission pattern contains link $j$. Denote by $N(i, j)$ the number of such patterns with a total number of active links equal to $i$. The probability $p(j)$ that link $j$ is active is

$$
p(j)=\sum_{i} N(i, j) \pi(i)
$$

and the fairness index can be computed using the definition of Section III-B2.

For $\rho>1, \pi(i)$ increases with the value of $i$, and the transmission patterns with a high number of active links have an increased probability of appearing, compared to those with only a few active links. In the limit $\rho \rightarrow \infty$, only the transmission patterns that maximize the spatial reuse have a nontrivial stationary probability. Consequently, when the average backoff time is much lower than the average exchange time, the idealized protocol achieves the maximal spatial reuse. However, all links that do not belong to the transmission patterns of maximal spatial reuse are starved.

The Line Topology: Equations (2) through (5) are valid for any network topology. Unfortunately, it is in general not possible to obtain a closed form expression for $N(i)$ nor $N(i, j)$. However, we can do so for a line topology, where $L+1$ nodes (numbered from 0 to $L$ ) are equally spaced (by 1 space unit) along a straight line (Fig. 6).

We define the parameter $l$ as 1 plus the minimal distance separating two noncolliding transmissions. This means that there can be an active link every $l$ space units. For example, if the RXRange and the CSRange cover exactly one neighbor, transmissions can take place simultaneously every 3 space units (see Fig. 6), so that $l=3$ in this case. Therefore, finding possible transmission patterns boils down to packing segments of length $l$ in a line interval of length $L+(l-1)$ (the additional term 


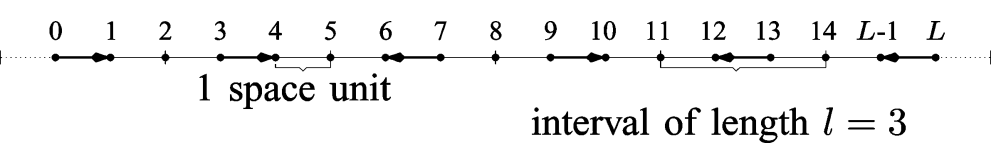

Fig. 6. A transmission pattern of maximal spatial reuse with symmetric exclusion domains (both RXRange and CSRange covering one neighbor).

$(l-1)$ compensates for the border effects, and is illustrated by the dashed segments in Fig. 6). If we place $i$ such segments, there remains $v=L-(l-1)-i l$ vacant units in the interval. The number of ways one can dispose these $i$ segments is thus equal to $i+v$ choose $i$. Finally, as each segment of length $l$ correspond to two possible link activations in the network (due to the symmetry assumption, each link and its reverse link occupy the same space), each possible arrangement of $i$ segments corresponds to $2^{i}$ possible link activation patterns, and we get

$$
N(i)=2^{i}\left(\begin{array}{c}
i+v \\
i
\end{array}\right)
$$

Next, we count the number of valid transmission patterns with $i$ active links that include a given link. We denote by $j_{\text {right }}$ the link between node $j-1$ and node $j$, and by $j_{\text {left }}$ the link between node $j$ and node $j-1$. To compute $N\left(i, j_{\text {right }}\right)$, we have to count the number of ways to place $i-1$ active links around the already active link $j_{\text {right }}$. Assume that we place $k$ of these active links on the left of link $j_{\text {right }}$, and $(i-1)-k$ on its right. We obtain

$$
\begin{aligned}
N\left(i, j_{\text {right }}\right)=2^{i-1} \sum_{k=0}^{i-1}\left(\begin{array}{c}
k+v_{\text {left }} \\
k
\end{array}\right) & \\
& \times\left(\begin{array}{c}
(i-1)-k+v_{\text {right }} \\
(i-1)-k
\end{array}\right)
\end{aligned}
$$

where $v_{\text {left }}=j-1-l k$ and $v_{\text {right }}=L-j-l((i-1)-k)=$ $L+l-j-l(i-k)$. Clearly, as the exclusion domain of link $j_{\text {right }}$ and link $j_{\text {left }}$ are identical by assumption, the computation of $N\left(i, j_{\text {left }}\right)$ yields the same result. By (5), this implies that, in the symmetric setting, two links in opposite directions have the same probability to be active.

Plugging the expressions for $N(i)$ and $N(i, j)=$ $N\left(i, j_{\text {right }}\right)=N\left(i, j_{\text {left }}\right)$ in (2) through (5) we can compute analytically the spatial reuse and the fairness index of the protocol on a finite line topology. Note that these values depend on the total length of the network $L$.

Theorem 1: For large line networks, i.e., when $L \rightarrow \infty$, the average spatial reuse $\sigma$ of the protocol under symmetric exclusion domains converges to

$$
\lim _{L \rightarrow \infty} \sigma=\frac{2 \rho y_{1}^{l-1}}{1+2 l \rho y_{1}^{l-1}}
$$

where $y_{1}$ is the positive real root of $1-y-2 \rho y^{l}$.

Proof: The result is obtained by applying Lemma 1 (in the Appendix) with $k=\left\lfloor\frac{L+(l-1)}{l}\right\rfloor, m=l, r=2 \rho$ and $n=$ $(L+(l-1)) \bmod l$. We then divide by $l$ to obtain the average spatial reuse $\sigma$.

Alternatively, this theorem can also be shown by adapting the approach of [37] which uses multivariate generating functions combined with the residue method. Although this approach yields the same result, we believe our approach is simpler.
Moreover, Lemma 1 can also be used to obtain the average spatial reuse under asymmetric exclusion domains, as we will see in the next section. As expected, when $\rho$ increases, $\sigma$ tends to $1 / l$, which is the maximal spatial reuse.

\section{Simulation Results}

The plain curves of Figs. 7 and 8 show the performance of the idealized protocol on the 50 node line network topology described in Section III-C ${ }^{2}$ with symmetric exclusion domains. Our analytical predictions and the values obtained by simulation differ by at most $0.2 \%$. Figs. 7 and 8 clearly illustrate the trade-off between spatial reuse and fairness. As $\rho$ increases (i.e., as the average backoff time becomes small compared to the average exchange time), the spatial reuse of the protocol reaches the maximal spatial reuse of 0.3469 (the value is slightly above $1 / 3$ because of the border effects) but the fairness decreases. Fig. 9(a) gives the probability that a link is active during the simulation. We see that only the links that belong to the pattern of maximal spatial reuse have a good access to the channel. In fact, our analysis tells us that in the limit, when $\rho \rightarrow \infty$, only these links have a nontrivial probability of accessing the channel: This means that, like the spatial reuse, the fairness index converges to 0.3469 . To summarize, under symmetric exclusion domains, a large fraction of the links ( $\simeq 2 / 3$ in our case) can be completely starved ${ }^{3}$.

\section{The Asymmetric Case With Full Capture EfFect}

\section{A. Specific Assumptions}

The exclusion domain around a link is asymmetric if there are nodes inside the transmitter's CSRange that are outside its RXRange (as Node 1 in Fig. 3). In our model, these nodes are unable to decode the RTS packets from the transmitter and are silenced only through the physical carrier sensing. Therefore, more nodes are silenced on the transmitter's side, and this is why we call the exclusion domain asymmetric.

As described in Section III-A4, if the set of nodes covered by the emitter's RXRange and CSRange differs, transmissions may overlap and create a capture effect. In this section, we assume that capture occurs whenever a node receives concurrently a strong signal from an emitter in its RXRange and a weaker signal from another emitter in its CSRange (but not in its RXRange). In particular, we do not take the order of arrival of the signals into account: even if the strongest signal begins after the weakest one, the receiver is able to synchronize on the strongest signal (see Fig. 10 for an example). This is a rather optimistic scenario, although it is supported to some extent by

\footnotetext{
${ }^{2}$ The impatient reader will have to wait until Section VI to discover the results on the 2-D topology as the line topology makes it easier to visualize and explain the behavior of the protocol.

${ }^{3}$ More detailed simulation studies under this setting have been performed in [3]. These studies show in particular that the above observations remain valid for other backoff and exchange time distributions.
} 


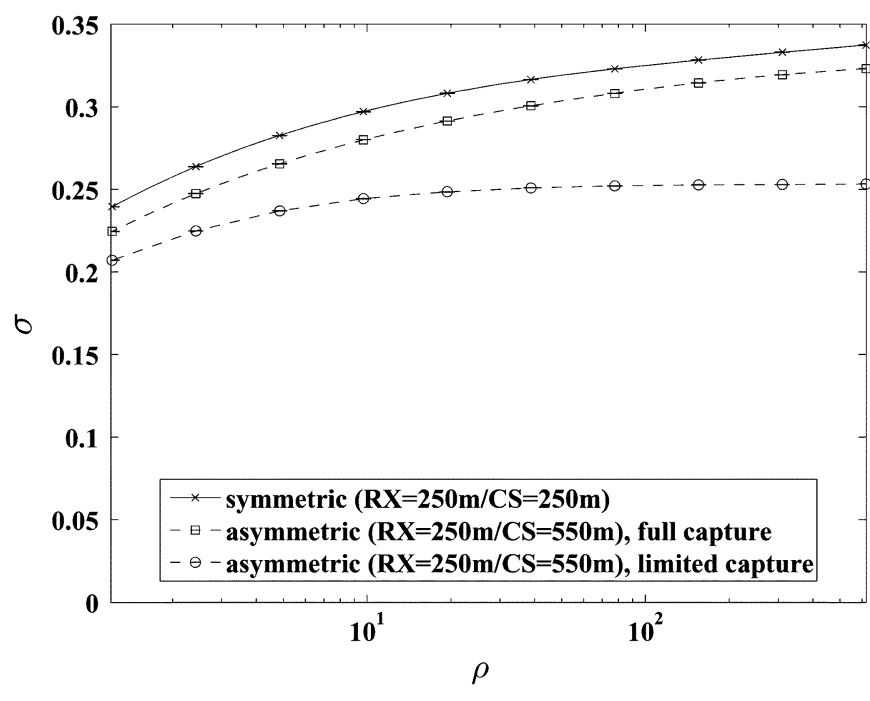

Fig. 7. Spatial reuse achieved by the idealized protocol on a line topology of 50 nodes, as a function of the access intensity $\rho$. The markers correspond to the results obtained by simulations and the curves to the results obtained analytically (except for the 'asymmetric $(\mathrm{RX}=250 \mathrm{~m} / \mathrm{CS}=550 \mathrm{~m})$, limited capture' case where we do not have the exact analytical curve).

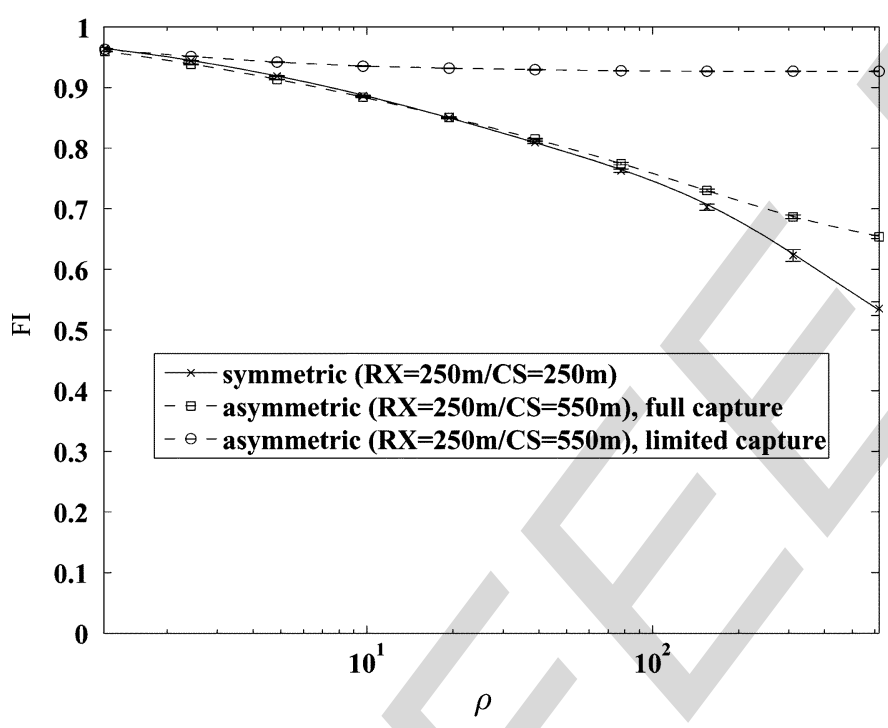

Fig. 8. Fairness index of the idealized protocol on a line topology of 50 nodes. The format is the same as in Fig. 7.

experimental evidences. [38] reports that capture occurs even if the strongest packet arrives later, but within the physical preamble of the first packet. In essence, full capture means that the order of arrival of the transmissions does not matter and it implies that patterns are valid independently of the order of arrival of the transmissions. Note that this last assumption will be removed in Section VI.

As an example, consider again a line network topology where nodes are equally spaced and the RXRange covers one neighbor, but where the CSRange covers two neighbors. A transmission pattern is valid if two consecutive receivers are separated by at least 2 space units, and if two consecutive transmitters are separated by at least 3 space units (see Fig. 11). Under this setting, the maximal spatial reuse is still $1 / 3$, but it is not possible to

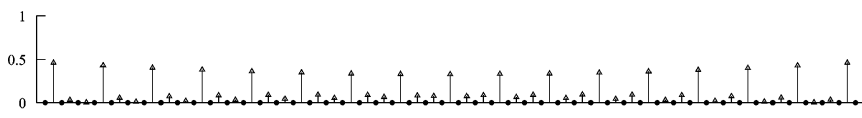

(a)



(b)

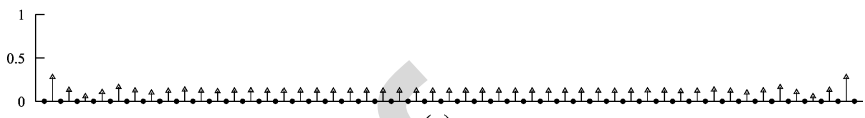

(c)

Fig. 9. On the $x$ axis, a line topology of 50 nodes. To each link $j_{\text {right }}$ (going from left to right) we associate a vertical bar that corresponds to the probability $p\left(j_{\text {right }}\right)$ that the link be active in the simulation for $\rho=620$ (the $p\left(j_{\text {left }}\right)$ can be obtained flipping the graphs). (a) Symmetric exclusion domain (RXRange $=$ CSRange $=250 \mathrm{~m}$ ). $\sigma=0.34, \mathrm{FI}=0.53$. (b) Asymmetric exclusion domains (RXRange $=250 \mathrm{~m}$, CSRange $=550 \mathrm{~m}$ ) with full capture. $\sigma=0.32, \mathrm{FI}=0.65$. (c) Asymmetric exclusion domains (RXRange $=$ $250 \mathrm{~m}$, CSRange $=550 \mathrm{~m}$ ) with limited capture. $\sigma=0.25, \mathrm{FI}=0.93$.

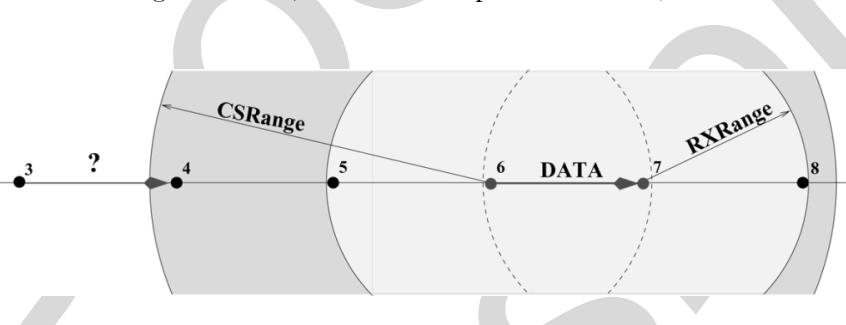

Fig. 10. Assume there is an ongoing DATA transmission between Node 6 and Node 7. As Node 4 is in the CSRange of Node 6, it can detect this transmission. If Node 3 sends a RTS to Node 4 we have two possibilities. Either Node 4 can resynchronize on this stronger signal, in which case the RTS will be followed by a complete exchange between Node 3 and Node 4; or it cannot resynchronize on the strongest signal and the RTS will be lost. In the full capture model, we always assume the first possibility whereas in the the limited capture model, the second possibility is assumed. Note that in the full capture model, a node that is silenced only through its physical carrier sensing (such as Node 4) can play the role of a receiver but it cannot initiate an exchange.

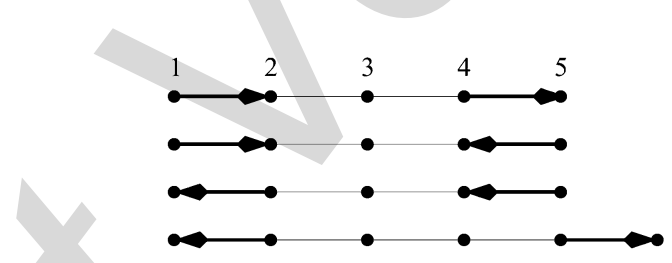

Fig. 11. Among the four possible configurations of two active links placed as closely to each other as possible (with $l=3$ ), only the three first ones maximize spatial reuse.

have two transmitters back to back in a pattern of maximal spatial reuse.

\section{B. Markov Modeling}

Asymmetric exclusion domains and capture effects make the analytical study of the CSMA/CA protocols more challenging and no model has been proposed so far. We now show that the Markovian framework of the previous section can be extended to include this case. The structure of the Markov chain remains essentially unchanged but some states (and the corresponding transitions) disappear. Take for example the Markov chain of Fig. 5 and assume that the CSRange now covers two neighbors instead of one. All transmission patterns remain valid except the 


\section{Direction change}

Fig. 12. A transmission pattern of maximal spatial reuse (with $l=3$ ). Active links are sorted in two groups, inside which all links point in the same direction.

\section{Vacant space}

Fig. 13. A transmission pattern with one vacant space (with $l=3$ ). There are three points where links change direction.

pattern 2.3 at the top level of the Markov chain, where two transmitters are back to back. This change in the set of valid transmission patterns does not affect the reversibility of the Markov chain. Equations (2) through (5) still hold, and the asymptotic properties of the protocol for $\rho \rightarrow \infty$ are thus unchanged. However, the number $N(i)$ of valid transmission patterns with $i$ active links and the number $N(i, j)$ of such patterns including link $j$ have to be recomputed. Again, this is only tractable for the simple topologies. We compute these numbers explicitly for the line topology in the following paragraph.

The Line Topology: We begin with the observation that two consecutive active links along the line can be separated by the least number of space units if and only if their orientation is such that the two transmitters are not back to back (see Fig. 11). Again, to each active link we associate an interval of length $l$ on the line. Consider a transmission pattern of maximal spatial reuse and assume that, once we have placed the intervals corresponding to its active links, there is no vacant space ${ }^{4}$; because of the above observation, all active links must point in the same direction until a certain point, and then point to the other direction (see Fig. 12). If $i$ is the number of active links, there are clearly $i+1$ possible settings. Now, let us assume that there is one vacant space. This vacant space will allow for an additional change of direction, as depicted in Fig. 13. Therefore, in this case, there is a total of three points where links change direction and there are

$$
\left(\begin{array}{c}
i+3 \\
3
\end{array}\right)=\left(\begin{array}{c}
i+3 \\
i
\end{array}\right)
$$

ways to pick these three points 5 . This reasoning can be extended to an arbitrary number of vacant spaces $v$, and we find

$$
N(i)=\left(\begin{array}{c}
i+2 v+1 \\
i
\end{array}\right)
$$

where $v=L+(l-1)-l i$.

To obtain an expression for $N(i, j)$ we follow the same procedure as in Section IV-B. If link $j_{\text {right }}$ from node $j-1$ to node $j$ is active, we enumerate the possible ways to place $k$ active links on its left side and $(i-1)-k$ active links on its right side. On the right side, the problem consists simply in packing $(i-1)-k$ intervals of length $l$ in an interval of length $L-j$. On the left

\footnotetext{
${ }^{4}$ Note that this is only possible for certain values of line size $L$.

${ }^{5}$ Note that these points can be collocated. Two collocated points correspond to a vacant space without a change of direction. Three collocated points correspond to a vacant space and two receivers facing each other. The problem is equivalent to counting the number of 3 -combinations with repetition of an $(i+1)$-element set.
}

side, the problem is slightly trickier, as node $j-l$ cannot emit, which means that in our previous reasoning we can do one less change of direction. We obtain

$$
\left(\begin{array}{c}
k+2 v_{\text {left }} \\
k
\end{array}\right)
$$

possible settings only, where $v_{\text {left }}=j-1-l k$. Combining the left and right parts, we get

$$
\begin{aligned}
N\left(i, j_{\text {right }}\right)=\sum_{k=0}^{i-1}\left(\begin{array}{c}
k+2 v_{\text {left }} \\
k
\end{array}\right) & \\
& \times\left(\begin{array}{c}
(i-1)-k+2 v_{\text {right }}+1 \\
(i-1)-k
\end{array}\right)
\end{aligned}
$$

where $v_{\text {right }}=L-j-l((i-1)-k)=L+l-j-l(i-k)$. The same reasoning allows to compute the value of $N\left(i, j_{\text {left }}\right)$ :

$$
\begin{aligned}
N\left(i, j_{\text {left }}\right)=\sum_{k=0}^{i-1}\left(\begin{array}{c}
k+2 v_{\text {left }}+1 \\
k
\end{array}\right) & \times\left(\begin{array}{c}
(i-1)-k+2 v_{\text {right }} \\
(i-1)-k
\end{array}\right) .
\end{aligned}
$$

Note that in the asymmetric case with full capture links $j_{\text {left }}$ and $j_{\text {right }}$ do not necessarily have the same probability to be active.

We can then plug the expressions for $N(i)$ and $N(i, j)$ in (2)(3)(4) through (5) to obtain the exact curve for the spatial reuse and the fairness index of the protocol.

The asymptotic spatial reuse for large networks is obtained in a similar way as in the case of symmetric exclusion domains.

Theorem 2: For line networks with $L \rightarrow \infty$, the average spatial reuse $\sigma$ of the protocol under asymmetric exclusion domains and full capture converges to

$$
\lim _{L \rightarrow \infty} \sigma=\frac{2 \rho y_{1}^{2 l-1}}{1+2 l \rho y_{1}^{2 l-1}}
$$

where $y_{1}$ is the positive real root of $1-y-\rho y^{2 l}$.

Proof: The result is obtained by applying Lemma 1 (in the Appendix) with $k=\left\lfloor\frac{L+(l-1)}{l}\right\rfloor, m=2 l, r=\rho$ and $n=$ $2((L+(l-1)) \bmod l)+1$. We then divide by $l$ to obtain the average spatial reuse $\sigma$.

Again, we verify that $\sigma$ tends indeed to $1 / l$ when $\rho$ tends to infinity.

\section{Simulation Results}

We illustrate by simulation the influence of asymmetric exclusion domains on the performance of the idealized protocol. Fig. 7 shows that its spatial reuse increases less rapidly than in the symmetric case. At a given $\rho$, the protocol with asymmetric exclusion domains has therefore on average fewer active links than in the symmetric case. This has a positive effect on the fairness of the protocol, especially at high values of $\rho$, where operating with a slightly lower number of active links greatly improves the fairness. Fig. 9(b) confirms that the starvation phenomenon is less pronounced than in the symmetric case and that the links in the middle of the line topology obtain a fairer access to the channel. Yet, we also see that the distribution of 
the link activity is not symmetric anymore. This is in agreement with our analysis that tells us that two links in opposite directions do not necessarily have the same probability of accessing the channel. This effect has a negative impact on the fairness index of the protocol. We have therefore two competing effects. Fig. 8 shows that, at low values of $\rho$, the two effects compensate each others; the fairness index of the protocol is very similar under symmetric and asymmetric exclusion domains. However, at high values of $\rho$, the positive effect dominates; the fairness index of the protocol in the asymmetric case with full capture is above its fairness index in the symmetric case. In the limit, when $\rho \rightarrow \infty$, the behavior of the protocol under asymmetric exclusion domains with full capture is essentially the same as under symmetric exclusion domains. The spatial reuse goes to 0.3469 and approximately $2 / 3$ of the links get starved. The only difference is that under asymmetric exclusion domains some links belong to more patterns of maximal spatial reuse as others, depending on their direction: this reduces the FI from 0.3469 to 0.2676 .

\section{The Asymmetric CASE With Limited CAPTURE EFFECT}

\section{A. Specific Assumptions}

In this section, we consider asymmetric exclusion domains with limited instead of full capture. Limited capture means that the capture effect occurs only if the strongest signal comes first. This assumption reflects the fact that in practice, many radio circuits cannot resynchronize to a stronger signal if they are already locked on another (weaker) carrier. Consequently, in this capture model, the order of arrival of the transmissions does matter.

Consider the example in Fig. 10. The transmission from Nodes 6 to 7 begins first. Node 4 senses this first transmission and locks its synchronization circuit on it. When Node 3 sends a RTS packet to initiate a transmission, Node 4 is unable to resynchronize and decode it. The RTS packet is lost and no transmission follows. As a result, in Fig. 11, the first (top) case is possible only if the left most link becomes active first (the opposite is true for the third case).

The limited capture effect is implemented in the most wellknown network simulators, including Ns-2 and Qualnet.

\section{B. Simulation Results}

These simulation results were at first quite surprising to us. Figs. 7 and 8 show the performance of the idealized protocol with asymmetric exclusion domains and limited capture. The spatial reuse of the protocol increases to a value much below $1 / 3$ but the fairness index of the protocol remains above 0.9 , even for large values of $\rho$. The access probabilities of the different links presented in Fig. 9(c) illustrate even better the dramatic improvement of the protocol fairness at high access intensity. Except at the borders, the situation is now completely fair. These results are good news in practice: They show that the starvation phenomenon is fixed by receivers having a limited capture capability, without requiring any other modification to the protocol.

To consolidate our findings, we show by simulation that this observation also holds in a random two-dimensional topology.

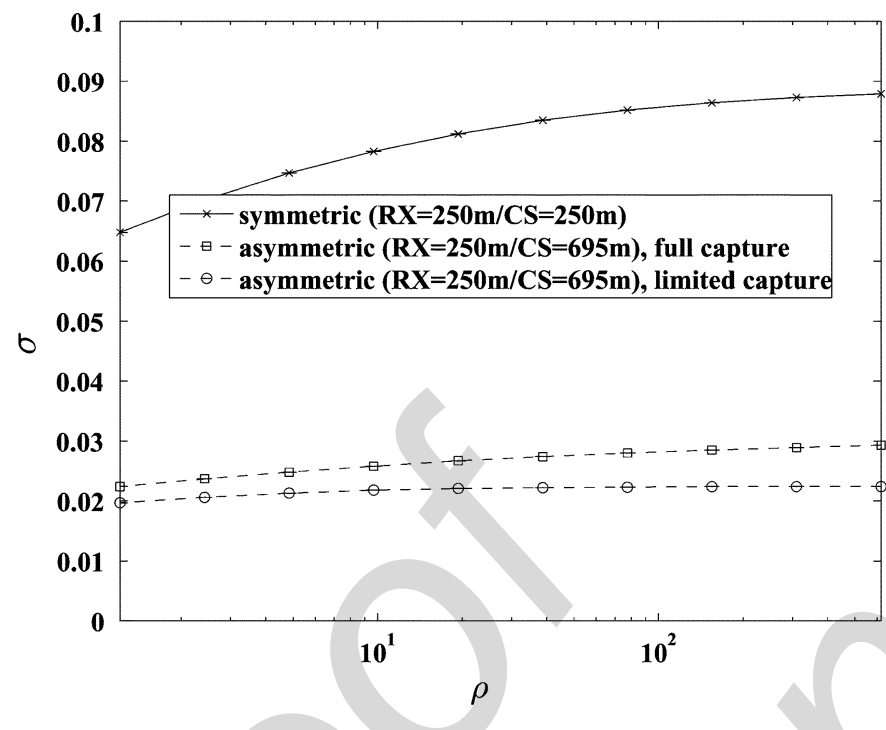

Fig. 14. Spatial reuse achieved by the idealized protocol on the 2-D topology (simulation results). The average spatial reuse is computed using the definition of Section III-B1 with $L=2435$. In this particular topology, at most 217 links can be activated simultaneously, yielding a maximal spacial reuse of 0.089 .

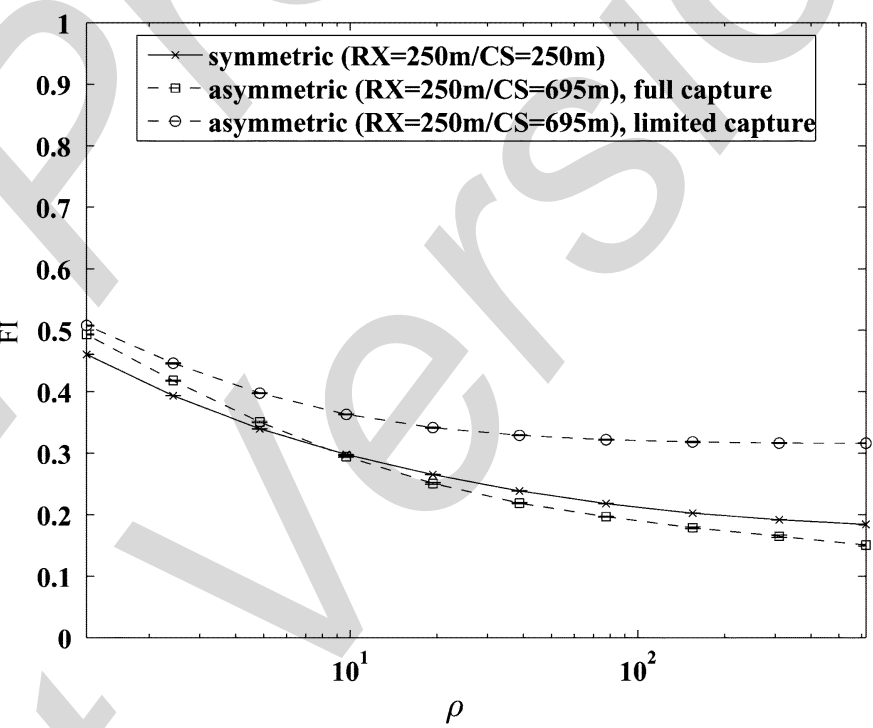

Fig. 15. Fairness index of the idealized protocol on the 2-D topology (simulation results).

The spatial reuse and fairness are reported in Fig. 14 and 15 respectively (the details of the simulation settings are described in Section III-C). Our study of the line topology showed that in the asymmetric case with limited capture, border effects can spread 5 hops away from the border; it was thus important to generate a sufficiently large topology to avoid that the performance of the protocol merely reflects the border effects. Now, a random topology has an inherent source of unfairness that is absent from regular topologies such as the line and is simply caused by the varying number of neighbors of each node. It explains why even at very low values of the access intensity $\rho$ the fairness index is not close to 1, contrary to the line. Despite this "topological unfairness" (which would be present not only in CSMA/CA protocols but also in any access protocol that does not adapt explicitly to the varying node degree), we see that in 


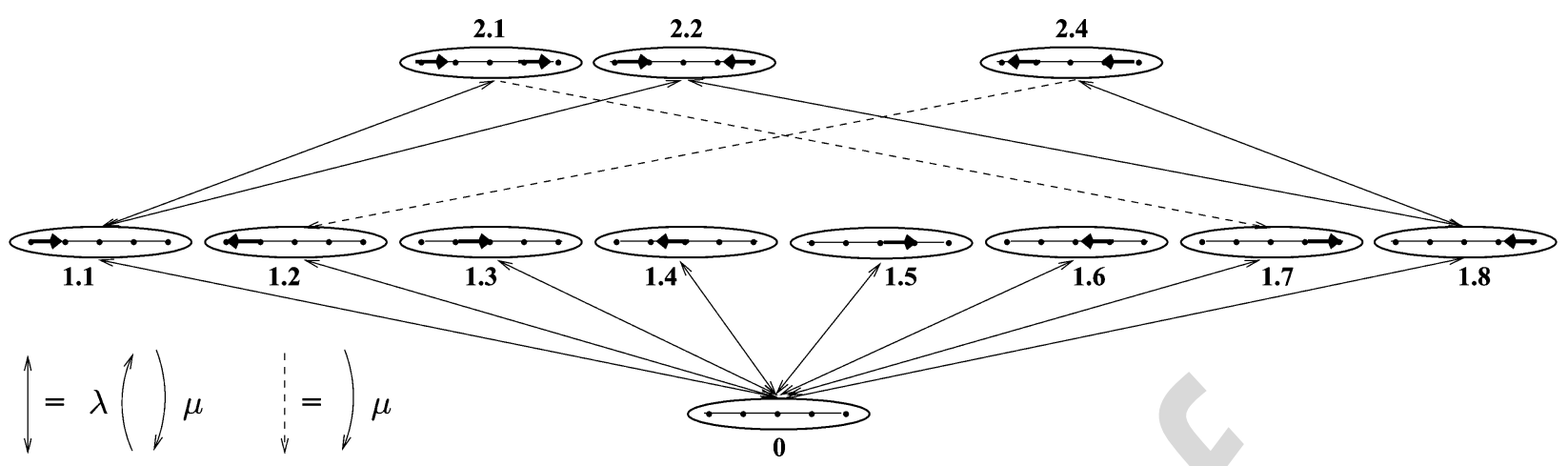

Fig. 16. The nonreversible Markov chain used to model the idealized protocol under asymmetric exclusion domains and limited capture.

this topology as well, the protocol with asymmetric exclusion domains and limited capture is the fairest when the value of $\rho$ becomes large.

\section{Markov Modeling}

The set of the valid transmission patterns (and thus the state space) is unchanged from the case with full capture effect. However, some states can now be reached only from a restricted set of lower states. Fig. 16 illustrates the Markov chain used to model the idealized protocol with limited capture effect. We see that, compared to the original Markov chain, two transition arrows have been removed: it accounts for the fact that the state at the top level can only be reached if the links become active in the right order.

Removing possible transitions between the states of the Markov chain breaks its regular structure, and the chain looses its reversibility property. This makes the analytical study of the chain very difficult as its stationary distribution does not have a nice product form anymore. However, a careful observation of the structure of the chain allows for the explanation of the results observed by simulation.

Let us first look back at the reversible case (corresponding to the two previous sections). For large values of $\rho$, the chain spends most of its time in the states of maximal spatial reuse. Indeed, when a transition occurs from a state at the top level to a state at the lower level (i.e., when an active link becomes idle), as $\lambda \gg \mu$, the next transition is most likely the reactivation of the same link, bringing the chain back to the same top level state. This explains why the protocol almost achieves maximal spatial reuse in the reversible case.

In the nonreversible case, this cannot happen anymore, because many of the transitions from top level states to lower level states cannot be reverted (see Fig. 16). Therefore, if the chain leaves a top level state, it might have to go down two or more levels before it can climb back to a top level state. The main consequence of this new dynamic is that the time spent in nonmaximal spatial reuse states (states below the top level) becomes nonnegligible.

In Proposition 1, we show that some states below the top level have a nontrivial (i.e., nonzero) stationary probability when $\rho \rightarrow$ $\infty$, contrary to the two previous cases. As a consequence, the average spatial reuse does no longer tend to the optimal value for increasing $\rho$.
Proposition 1: There exist transmission patterns of nonmaximal spatial reuse with a strictly positive stationary probability when $\rho \rightarrow \infty$.

Proof: Consider a transmission pattern $x^{*}$ of maximal spatial reuse, and another pattern $x^{\prime}$ obtained by removing an active link from $x^{*}$ and such that there is no possible transition (in the Markov chain) from $x^{\prime}$ to $x^{*}$ (which is only possible in the limited capture case). Assume furthermore that $x^{\prime}$ has $i$ active links. Now, the balance equation for $x^{\prime}$ reads

$$
\pi_{x^{\prime}} i \mu=\pi_{x^{*}} \mu+\sum_{y \in A_{x^{\prime}}} \pi_{y} \lambda
$$

where $A_{x^{\prime}}$ denotes the set of the states $y$ at level $i-1$ such that a transition from $y$ to $x^{\prime}$ is possible. Dividing both sides of this equation by $i \mu$, we obtain that $\pi_{x^{\prime}} \geq \pi_{x^{*}} / i$. Therefore, when $\rho$ tends to infinity, if $x^{*}$ has a non trivial asymptotic stationary probability, then so does $x^{\prime}$.

However, the loss in the spatial reuse is compensated by an increase in the fairness of the protocol. Indeed, contrary to the two previous cases, links that do not belong to a pattern of maximal spatial reuse do not necessarily get starved.

Proposition 2: There exist links that do not belong to the patterns of maximal spatial reuse but have a strictly positive probability of being active when $\rho \rightarrow \infty$.

Proof: Take a state $x^{\prime}$ as above. Remove one additional active link (next to the one removed to obtain $x^{\prime}$ from $x^{*}$ ) to obtain state $y$. From state $y$ it is now possible to reach a state $z$ that contains a link that does not belong to any patterns of maximal spatial reuse. We now show that this state has a nontrivial probability. Using the balance equations, we have

$$
\pi_{y}\left(\mu(i-1)+\lambda\left|A_{y}\right|\right)>\mu \pi_{z}+\mu \pi_{x^{\prime}}
$$

where $\left|A_{y}\right|$ is the number of states at level $i$ connected to $y$. As

$$
\pi_{z} i \mu>\lambda \pi_{y}>\frac{\lambda}{\mu(i-1)+\lambda\left|A_{y}\right|}\left(\mu \pi_{z}+\mu \pi_{x^{\prime}}\right)
$$

we obtain

$$
\pi_{z}\left(\mu i(i-1)+\lambda i\left|A_{y}\right|-\lambda\right)>\lambda \pi_{x^{\prime}}
$$


which implies that for $\rho \rightarrow \infty$

$$
\pi_{z}>\frac{1}{i\left|A_{y}\right|-1} \pi_{x^{\prime}}
$$

Therefore, if $x^{\prime}$ has a nontrivial asymptotic stationary probability, so does $z$, and hence there are links that do not belong to the patterns of maximal spatial reuse but have a strictly positive probability of being active when $\rho \rightarrow \infty$.

Proposition 2 implies that, on the line topology, the starvation effect can always be avoided. In addition, we can show that in the asymmetric case with limited capture links in opposite direction have the same probability of being active (this was not the case under full capture). Indeed given any transmission pattern, a link can become active if and only if there is no transmitting node in the carrier sensing range of its two end nodes. Thus, if link $j_{\text {left }}$ can become active so can link $j_{\text {right }}$.

Note that Propositions 1 and 2 are not restricted to the line topology and can be applied to any network topology provided that the corresponding Markov chain includes states of type $x^{*}$, $x^{\prime}, y$, and $z$.

\section{CONCLUSION}

In multihop ad hoc networks, CSMA/CA protocols can create a high level of unfairness among the network links. This unfairness appears even on the simplest regular network topologies where all nodes, except the ones at the border, have the same number of neighbors.

In this paper, we introduce a class of continuous Markov chain models which explains accurately this observation but also shows that the performance of these protocols vary with the capture and sensing capabilities of the network nodes.

On the positive side, we find that for a given (finite) access intensity $\rho, \mathrm{CSMA} / \mathrm{CA}$ protocols are fairer when the receiving and carrier sensing ranges are significantly different (asymmetric case) than when they have virtually the same values (symmetric case). On the negative side, we show that the price to pay for this higher fairness is a lower spatial reuse.

In addition, we demonstrate that, when $\rho \rightarrow \infty$, the capture capabilities of the network nodes play a decisive role on the performance of the protocol. In the asymmetric case with full capture (as well as in the commonly adopted symmetric case), the spatial reuse is maximal but all the links that do not belong to the patterns of maximal spatial reuse are starved. In contrast, in the asymmetric case with limited capture, the spatial reuse is not maximal but starvation can be avoided.

\section{APPENDIX}

In this Appendix, we establish the following lemma, which we use to prove Theorems 1 and 2 .

Lemma 1: Let $\{X(k), k \geq 1\}$, be an infinite sequence of discrete random variables defined by their distribution

$$
= \begin{cases}Z^{-1} r^{i}\left(\begin{array}{c}
k m-(m-1) i+n \\
i
\end{array}\right), & 0 \leq i \leq k \\
0, & \text { otherwise }\end{cases}
$$

where $m$ and $n$ are fixed finite integer parameters with $m \geq 2$ and $n \geq 0, r$ is a positive real number, and where

$$
Z=\sum_{i=0}^{k} r^{i}\left(\begin{array}{c}
k m-(m-1) i+n \\
i
\end{array}\right)
$$

is a normalizing constant. Then

$$
\lim _{k \rightarrow \infty} \frac{\mathbb{E}(X(k))}{k}=\frac{m r y_{1}{ }^{m-1}}{1+m r y_{1}{ }^{m-1}}
$$

where $y_{1}$ is the positive real root of $1-y-r y^{m}$.

Theorems 1 and 2 are two particular cases of this lemma, where the random variables $X(k)$ are the levels of the Markov chain (with $k=\lfloor(L+l-1) / l\rfloor$ ), and whose invariant distribution $N(i) \pi(i)$ coincides with the distribution $p_{k}(i)$ in the lemma, for particular values of $m, r$ and $n$ that differ for both theorems. As a result, the asymptotic average spatial reuse for large $L$ (and thus $k$ ) is given by (6), for the appropriate values of $m, r$ and $n$.

\section{A. Outline of the Proof of Lemma 1}

The proof can be subdivided in three main steps:

1) we first show that there is an integer $0<i_{k}^{*}<k$ such that $p_{k}\left(i_{k}^{*}\right)$ is maximal;

2) we then show that

$$
\lim _{k \rightarrow \infty} \frac{\mathbb{E}\left(X(k)-i_{k}^{*}\right)}{k}=0 .
$$

This implies that $\lim _{k \rightarrow \infty} \frac{\mathbb{E}(X(k))}{k}$ exists if and only if $\lim _{k \rightarrow \infty} \frac{i_{k}^{*}}{k}$ exists;

3) the last step of the proof consists in computing this limit, and showing that it is (6).

\section{B. Computation}

1) Step 1: We first show that the function $p_{k}(i)$ is maximum for some $0<i_{k}^{*}<k$, by using functions of the ratios of these quantities. We define the functions $\alpha_{k}(i)$ for $1 \leq i \leq k$ as

$$
\begin{aligned}
\alpha_{k}(i) & \doteq \frac{p_{k}(i)}{p_{k}(i-1)} \\
& =r \frac{\left(\begin{array}{c}
k m-(m-1) i+n \\
i
\end{array}\right)}{\left(\begin{array}{c}
k m-(m-1)(i-1)+n \\
i-1
\end{array}\right)} \\
& =\frac{r(k m-m(i-1)+n)_{m}}{i(k m-(m-1)(i-1)+n)_{m-1}}
\end{aligned}
$$

where the notation $(x)_{m}$ stands for $(x)_{m}=\prod_{j=0}^{m-1}(x-j)$. Therefore, the ratio between two consecutive terms is

$$
\begin{aligned}
\frac{\alpha_{k}(i+1)}{\alpha_{k}(i)}=\frac{i}{i+1} \cdot \frac{(k m-m i+n)_{m}}{(k m-m(i-1)+n)_{m}} & \frac{(k m-(m-1)(i-1)+n)_{m-1}}{(k m-(m-1) i+n)_{m-1}}
\end{aligned}
$$

$p_{k}(i) \doteq \mathbb{P}(X(k)=i)$ 
which can be rewritten as

$$
\begin{aligned}
& \frac{\alpha_{k}(i+1)}{\alpha_{k}(i)}=\frac{i}{i+1} \cdot \frac{k m-m i+n-m+1}{k m-m i+n+1} . \\
& \prod_{j=0}^{m-2} \frac{(k m-m i+n-j)(k m-(m-1)(i-1)+n-j)}{(k m-m(i-1)+n-j)(k m-(m-1) i+n-j)} .
\end{aligned}
$$

A close observation of each fraction allows to see that for $m>1$ each fraction is strictly smaller than one. Thus, $\alpha_{k}$ is a strictly decreasing function that ranges from $\alpha_{k}(1)=r((k-1) m+n+$ $1)>1$ to $\alpha_{k}(k)=r(m+n)_{m} /\left(k(k+m-1+n)_{m-1}\right)<1$. This implies that there is a value $0<i_{k}^{*}<k$ such that $\alpha_{k}\left(i_{k}^{*}+\right.$ $1) \leq 1$ and $\alpha_{k}\left(i_{k}^{*}\right)>1$. Consequently, $p_{k}\left(i_{k}^{*}\right)$ is maximal, and $\alpha_{k}\left(i_{k}^{*}+2\right)<1$.

2) Step 2: We now move on to the computation of the expected value of $X(k)$. We will see that the expected value is close to $i_{k}^{*}$ for large $k$; hence, we directly compute $\mathbb{E}\left(X(k)-i_{k}^{*}\right)$ instead of $\mathbb{E}(X(k))$. We also introduce the values $\hat{\imath}_{k}:=i_{k}^{*}+f(k)$ and $\check{\imath}_{k}:=i_{k}^{*}-f(k)$, where $f(k)$ is an integer function such that $\lim _{k \rightarrow \infty} f(k) / \sqrt{k}=0$ but $\lim _{k \rightarrow \infty} f(k) / \log k=\infty$, to decompose the summation in

$$
\mathbb{E}\left(X(k)-i_{k}^{*}\right)=\sum_{i=0}^{k}\left(i-i_{k}^{*}\right) p_{k}(i)
$$

in three terms

$$
\begin{aligned}
\mathbb{E}\left(X(k)-i_{k}^{*}\right) & =\sum_{i=0}^{\check{\iota}_{k}}\left(i-i_{k}^{*}\right) p_{k}(i) \\
& +\sum_{i=\check{\iota}_{k}+1}^{\hat{\imath}_{k}-1}\left(i-i_{k}^{*}\right) p_{k}(i)+\sum_{i=\hat{\imath}_{k}}^{k}\left(i-i_{k}^{*}\right) p_{k}(i)
\end{aligned}
$$

We show that each of these three terms is of order $o(k)$. For the first one, using the definition of $\alpha_{k}$ and the fact that it is a striclty decreasing function, we find that

$$
\begin{aligned}
0 \leq & \sum_{i=0}^{\check{\imath}_{k}}\left(i_{k}^{*}-i\right) p_{k}(i) \\
\leq & p_{k}\left(\check{\imath}_{k}\right) \sum_{i=0}^{\check{\iota}_{k}}\left(i_{k}^{*}-i\right) \\
\leq & p_{k}\left(\check{\imath}_{k}\right) \sum_{i=0}^{k}(k-i) \\
= & (k(k+1) / 2) p_{k}\left(\check{\imath}_{k}\right) \\
= & (k(k+1) / 2) p_{k}\left(i_{k}^{*}-1\right) \prod_{j=\check{\imath}_{k}+1}^{i_{k}^{*}-1}\left(\alpha_{k}(j)\right)^{-1} \\
= & (k(k+1) / 2) p_{k}\left(i_{k}^{*}-1\right) \\
& \quad \times \prod_{j=0}^{f(k)-2}\left(\alpha_{k}\left(i_{k}^{*}-1-j\right)\right)^{-1} \\
\leq & (k(k+1) / 2) p_{k}\left(i_{k}^{*}-1\right) \\
& \times\left(\alpha_{k}\left(i_{k}^{*}-1\right)\right)^{-f(k)+2} \\
\leq & (k(k+1) / 2)\left(\alpha_{k}\left(i_{k}^{*}-1\right)\right)^{-f(k)+2}
\end{aligned}
$$

where that last line follows from $p_{k}\left(i_{k}^{*}-1\right) \leq 1$. As $\alpha\left(i_{k}^{*}-1\right)>$ 1 , this expression tends to 0 when $k$ tends to infinity, which shows that the first term in $(8)$ is $o(k)$. The third term can be bounded in a similar manner, whereas the computation for the middle term reads

$$
\begin{aligned}
& \left|\sum_{i=\breve{\imath}_{k}+1}^{\hat{\imath}_{k}-1}\left(i-i_{k}^{*}\right) p_{k}(i)\right| \\
& \quad \leq \sum_{i=\check{\imath}_{k}+1}^{\hat{\imath}_{k}-1}\left|\left(i-i_{k}^{*}\right) p_{k}(i)\right| \\
& \quad=\sum_{i=-f(k)+1}^{f(k)-1}|i| p_{k}\left(i+i_{k}^{*}\right) \\
& \leq \sum_{i=-f(k)}^{f(k)} f(k) p_{k}\left(i+i_{k}^{*}\right) \\
& \leq(2 f(k)+1) f(k) \\
& =o(k) .
\end{aligned}
$$

Combining the three terms, we conclude that (8) is of order $o(k)$, and thus that

$$
\lim _{k \rightarrow \infty} \frac{\mathbb{E}\left(X(k)-i_{k}^{*}\right)}{k}=0
$$

which implies in turn that $\lim _{k \rightarrow \infty} \mathbb{E}(X(k)) / k$ exists if and only if $\lim _{k \rightarrow \infty} i_{k}^{*} / k$ exists.

3) Step 3: The last step of the proof thus consists in computing the latter limit. We first extend the support of the function $\alpha_{k}(i)$ from $\mathbb{N}$ to $\mathbb{R}^{+}$, by observing in (7) that $\alpha_{k}(i)$ is well defined for noninteger values of $i$. The function $\alpha_{k}(x)$ over $x \in \mathbb{R}^{+}$, is a continuous, strictly decreasing function, and we can therefore find the value $x \in \mathbb{R}$ such that $\alpha_{k}(x)=1$. Clearly, $\left|x-i_{k}^{*}\right|<1$, so that $\lim _{k \rightarrow \infty} x / k=\lim _{k \rightarrow \infty} i_{k}^{*} / k$. We need therefore to compute the root $x \in \mathbb{R}^{+}$of the equation $\alpha_{k}(x)=1$, which can be expanded from (7) as

$$
\frac{r \prod_{j=0}^{m-1}(k m-m(x-1)+n-j)}{x \prod_{j=0}^{m-2}(k m-(m-1)(x-1)+n-j)}=1 .
$$

Dividing the numerator and denominator by $k^{m}$, we obtain

$$
\begin{aligned}
r \prod_{j=0}^{m-1}(m-m & \left.\left(\frac{x}{k}-\frac{1}{k}\right)+\frac{n-j}{k}\right) \\
& =\frac{x}{k} \prod_{j=0}^{m-2}\left(m-(m-1)\left(\frac{x}{k}-\frac{1}{k}\right)+\frac{n-j}{k}\right) .
\end{aligned}
$$

Taking the limit of both sides for $k \rightarrow \infty$ we get

$$
r \prod_{j=0}^{m-1}\left(m-m \frac{x}{k}\right)=\frac{x}{k} \prod_{j=0}^{m-2}\left(m-(m-1) \frac{x}{k}\right),
$$

and solving for $r$, we obtain

$$
r=\frac{x}{m(k-x)}\left(1+\frac{x}{m(k-x)}\right)^{m-1} .
$$


Let

$$
y=\left(\frac{x}{m(k-x) r}\right)^{1 /(m-1)}
$$

or equivalently

$$
\frac{x}{k}=\frac{m r y^{m-1}}{1+m r y^{m-1}} .
$$

Using this change of variables in (9), we get

$$
r=r y^{m-1}\left(1+r y^{m-1}\right)^{m-1}
$$

which we can recast as

$$
1-y-r y^{m}=0 .
$$

By Descartes' rule of signs, the difference between the number of positive real roots of a real polynomial and the number of changes of signs of the sequence of its coefficients is always an even, non positive number. The polynomial $1-y-r y^{m}$ has one change of sign, and therefore only one positive real root (as we could expect since $\alpha(x)$ is continuous and strictly decreasing for $x \in \mathbb{R}^{+}$), which we denote by $y_{1}$.

Because of (10), we have therefore proven that

$$
\lim _{k \rightarrow \infty} \frac{\mathbb{E}(X(k))}{k}=\frac{m r y_{1}{ }^{m-1}}{1+m r y_{1}{ }^{m-1}}
$$

where $y_{1}$ is the real solution of (11).

\section{REFERENCES}

[1] IEEE802.11, Part 11: Wireless LAN Medium Access Control (MAC) and Physical Layer (PHY) Specifications Aug. 1999, IEEE Std..

[2] X. Wang and K. Kar, "Throughput modelling and fairness issues in CSMA/CA based ad-hoc networks," in Proc. INFOCOM, Miami, FL, 2005.

[3] M. Durvy and P. Thiran, "A packing approach to compare slotted and nonslotted medium access control," in Proc. INFOCOM, Barcelona, Spain, 2006.

[4] M. M. Carvalho and J. J. Garcia-Luna-Aceves, "A scalable model for channel access protocols in multihop ad hoc networks," in Proc. MobiCom, 2004, pp. 330-344, ACM Press.

[5] M. Garetto, T. Salonidis, and E. Knightly, "Modeling per-flow throughput and capturing starvation in CSMA multihop wireless networks," in INFOCOM, Barcelona, Spain, 2006.

[6] K. Medepalli and F. Tobagi, "Towards performance modeling of IEEE 802.11 based wireless networks: A unified framework and its applications," in Proc. INFOCOM, Barcelona, Spain, 2006.

[7] M. Garey and D. Johnson, Computers and Intractability: A Guide to the Theory of NP-Completeness. New York: Freeman, 1983.

[8] L. Tassiulas and A. Ephremides, "Stability properties of constrained queueing systems and scheduling for maximum throughput in multihop radio networks," IEEE Trans. Autom. Control, vol. 37, no. 12, pp. 1936-1949, 1992.

[9] E. Modiano, D. Shah, and G. Zussman, "Maximizing throughput in wireless networks via gossip," in Proc. ACM SIGMETRIC/Perform., 2006.

[10] K. Jung and D. Shah, "Low delay scheduling algorithms," in Proc. IEEE ISIT, 2007.

[11] G. Bianchi, "Performance analysis of the IEEE 802.11 distributed coordination function," IEEE J. Sel. Areas Commun., vol. 18, no. 3, 2000.

[12] F. Calì, M. Conti, and E. Gregori, "IEEE 802.11 protocol: Design and performance evaluation of an adaptive backoff mechanism," IEEE J. Sel. Areas Commun., vol. 18, no. 9, pp. 1774-1780, 2000.
[13] F. Calì, M. Conti, and E. Gregori, "Dynamic tuning of the IEEE 802.11 protocol to achieve a theoretical throughput limit," IEEE/ACM Trans. Netw., vol. 8, no. 6, pp. 785-799, 2000.

[14] M. Zorzi and R. Rao, "Capture and retransmission control in mobile radio," IEEE J. Sel. Areas Commun., vol. 12, no. 8, pp. 1289-1298, Oct. 1994.

[15] C. T. Lau and C. Leung, "Capture models for model packet radio networks," IEEE Trans. Commun., vol. 40, no. 5, pp. 917-925, May 1992.

[16] W. Luo and A. Ephremides, "Power levels and packet lengths in random multiple access," IEEE Trans. Inf. Theory, vol. 48, no. 1, pp. 46-58, Jan. 2002.

[17] Z. Hadzi-Velkov and B. Spasenovski, "On the capacity of IEEE 802.11 DCF with capture in multipath-faded channels," Int. J. Wireless Inf. Net., vol. 9, no. 3, pp. 191-199, July 2002.

[18] Z. Hadzi-Velkov and B. Spasenovski, "Capture effect with diversity in IEEE 802.11b DCF," in Proc. IEEE ISCC'03, Jun. 2003.

[19] H. S. Chhaya and S. Gupta, "Performance modeling of asynchronous data transfer methods of IEEE 802.11 mac protocol," Wirel. Netw., vol. 3, no. 3, pp. 217-234, 1997.

[20] N. Gupta and P. R. Kumar, "A performance analysis of the IEEE 802.11 wireless LAN medium access control," Commun. Inf. Syst., vol. 3, no. 4, pp. 279-304, 2003.

[21] Y. Wang and J. J. Garcia-Luna-Aceves, "Modeling of collision avoidance protocols in single-channel multihop wireless networks," Wirel. Netw., vol. 10, no. 5, pp. 495-506, 2004.

[22] F. A. Tobagi, "Modeling and performance analysis of multihop packet radio networks," Proc. IEEE, vol. 75, no. 1, pp. 135-155, 1987.

[23] R. Boorstyn, A. Kershenbaum, B. Maglaris, and V. Sahin, "Throughput analysis in multihop CSMA packet radio networks," IEEE Trans. Commun., vol. 35, no. 3, pp. 267-274, 1987.

[24] A. Proutiere, Y. Yi, and M. Chiang, "Throughput of random medium access control: An asymptotic approach," in Proc. CISS'08, Mar. 2008.

[25] A. Jindal and K. Psounis, "Achievable rate region and optimality of multihop wireless 802.11-scheduled networks," in Proc. ITA'08, Jan. 2008.

[26] C. Bordenave, D. McDonald, and A. Proutiere, "Performance of random medium access control: An asymptotic approach," in Proc. ACM Sigmetrics'08, June 2008.

[27] M. Hira, F. Tobagi, and K. Medepalli, "Throughput analysis of a path in an IEEE 802.11 multihop wireless network," in Proc. IEEE Wireless Commun. Netw. Conf. (WCNC 2007), Mar. 2007, pp. 441-446.

[28] L. T. Nguyen, R. Beuran, and Y. Shinoda, "Performance analysis of IEEE 802.11 in multihop wireless networks," Mobile Ad-Hoc and Sensor Networks, ser. Lecture Notes in Computer Science, vol. 4864, pp. 326-337, 2007.

[29] P. C. Ng and S. C. Liew, "Throughput analysis of IEEE 802.11 multihop ad hoc networks," IEEE/ACM Trans. Netw., vol. 15, no. 2, pp. 309-322, 2007.

[30] M. Durvy, O. Dousse, and P. Thiran, "Modeling the 802.11 protocol under different capture and sensing capabilities," in INFOCOM (Minisymposium), Anchorage, AK, 2007.

[31] M. Durvy and P. Thiran, "Understanding the gap between the IEEE 802.11 protocol performance and the theoretical limits," in Proc. SECON, Reston, VA, 2006.

[32] V. Bharghavan, A. Demers, S. Shenker, and L. Zhang, "MACAW: A media access protocol for wireless LAN's," in Proc. SIGCOMM, 1994 pp. 212-225, ACM Press.

[33] R. Jain, The Art of Computer Systems Performance Analysis. New York: Wiley, 1991.

[34] Discrete Event Simulator for the Idealized 802.11 Protocol, [Online] Available: http://icapeople.epfl.ch/mdurvy/research.html

[35] F. P. Kelly, "Loss networks," Ann. Appl. Probab., vol. 1, no. 3, pp. 319-378, 1991.

[36] D. Y. Burman, J. P. Lehoczky, and Y. Lim, "Insensitivity of blocking probabilities in a circuit-switching network," J. Appl. Probab., vol. 21 , no. 4, pp. 850-859, 1984.

[37] Y. Baryshnikov, E. G. Coffman Jr., and P. Jelenković, "Space filling and depletion," J. Appl. Probab., vol. 41, no. 3, pp. 691-702, 2004.

[38] A. Kochut, A. Vasan, A. Shankar, and A. Agrawala, "Sniffing out the correct physical layer capture model in 802.11b," in Proc. ICNP, 2004.

Mathilde Durvy (S'02) received the M.Sc. degree in 2002 and the Ph.D. degree in 2007 in communication systems from EPFL, Lausanne, Switzerland.

Her research interests are in the design and analysis of medium access control protocols for multihop ad hoc networks.

In 2005, she received an Infocom travel grant and in 2007, she was a finalist of the Google Anita Borg scholarship. 
Olivier Dousse (M'00) received the M.Sc. degree in physics from the Swiss Federal Institute of Technology at Lausanne, Switzerland (EPFL) in 2000, and the $\mathrm{Ph} . \mathrm{D}$. degree in communication systems from the same institution in 2005.

From 2006 to 2008, he was Research Scientist at Deutsche Telekom Laboratories in Berlin. He is currently a member of research staff at Nokia Research Center in Lausanne. His research interests are in stochastic models for communication networks.

Dr. Dousse received the honorable mention of the 2005 ACM Doctoral Dissertation Competition, and he was awarded the Top 3 Student Prize of the EPFL Graduate School in communication systems in 2001. He was runner-up for the IEEE-INFOCOM Best Paper Award in 2003.

Patrick Thiran (S'89-M'97) received the electrical engineering degree from the Université Catholique de Louvain, Louvain-la-Neuve, Belgium, in 1989, the M.S. degree in electrical engineering from the University of California at Berkeley, USA, in 1990, and the Ph.D. degree from EPFL, in 1996.

$\mathrm{He}$ is an Associate Professor at EPFL. He became an Adjunct Professor in 1998, an Assistant Professor in 2002 and an Associate Professor in 2006. From 2000 to 2001, he was with Sprint Advanced Technology Labs, Burlingame, CA. His research interests include communication networks, performance analysis, dynamical systems, and stochastic models. He is currently active in the analysis and design of wireless multihop networks and in network monitoring.

Dr. Thiran served as an Associate Editor for the IEEE TRANSACTIONS ON CIRCUITS AND SYSTEMS in 1997-1999, and he is currently an Associate Editor for the IEEE/ACM TRANSACTIONS ON NETWORKING. He was the recipient of the 1996 EPFL Ph.D. award. 